\title{
Cognitive Capacity Harvesting Networks: Architectural Evolution Toward Future Cognitive Radio Networks
}

\author{
Haichuan Ding, Yuguang Fang, Fellow, IEEE, Xiaoxia Huang, Member, IEEE, Miao Pan, Member, IEEE, \\ Pan Li, Member, IEEE, and Savo Glisic, Senior Member, IEEE
}

\begin{abstract}
Cognitive radio technologies enable users to opportunistically access unused licensed spectrum and are viewed as a promising way to deal with the current spectrum cri-sis. Over the last 15 years, cognitive radio technologies have been extensively studied from algorithmic design to practical implementation. One pressing and fundamental problem is how to integrate cognitive radios into current wireless networks to enhance network capacity and improve users' experience. Unfortunately, existing solutions to cognitive radio networks (CRNs) suffer from many practical design issues. To foster further research activities in this direction, we attempt to provide a tutorial for CRN architecture design. Noticing that an effec-tive architecture for CRNs is still lacking, in this tutorial, we systematically summarize the principles for CRN architecture design and present a novel flexible network architecture, termed cognitive capacity harvesting network (CCHN), to elaborate on how a CRN architecture can be designed. Unlike existing architectures, we introduce a new network entity, called secondary service provider, and deploy cognitive radio capability enabled routers, called cognitive radio routers, in order to effectively and efficiently manage resource harvesting and mobile traf-fic while enabling users without cognitive radios to access and
\end{abstract}

This work was supported in part by the U.S. National Science Foundation under Grant CNS-1343356, Grant CNS-1409797, and Grant CNS-1423165. The work of X. Huang was supported in part by the NSFC-Guangdong Joint Program under Grant U1501255 and Gran U1301256, in part by the Guangdong Science and Technology Project under Grant 2015A010103009, and in part by the Shenzhen Science and Technology Project under Grant CXZZ20150401152251212. The work of M. Pan was supported in part by the U.S. National Science Foundation under Gran CNS-1343361, Grant CNS-1350230, and Grant CPS-1646607. The work of

P. Li was supported by the U.S. National Science Foundation under Grant CNS-1602172 and Grant CNS-1566479. The work of S. Glisic was supported by the Taseen käitto CWC-NS-Glisic-Menot under Project 240007101. (Corresponding author: Yuguang Fang.)

H. Ding is with the Department of Electrical and Computer Engineering, University of Florida, Gainesville, FL 32611 USA (e-mail: dhcbit@gmail.com).

Y. Fang is with the Department of Electrical and Computer Engineering, University of Florida, Gainesville, FL 32611 USA, and also with Dalian Maritime University, Dalian, China (e-mail: fang@ece.ufl.edu).

$X$. Huang is with the Shenzhen Institutes of Advanced Technology, Chinese Academy of Sciences, Shenzhen 518000, China (e-mail: xx.huang@siat.ac.cn).

M. Pan is with the Department of Electrical and Computer Engineering, University of Houston, Houston, TX 77204 USA (e-mail: mpan2@uh.edu).

$\mathrm{P} . \mathrm{Li}$ is with the Department of Electrical Engineering and Computer Science, Case Western Reserve University, Cleveland, OH 44106 USA (e-mail: lipan@case.edu).

S. Glisic is with the Telecommunications Laboratory, University of Oulu, 90014 Oulu, Finland (e-mail: savo.glisic@ee.oulu.fi). enjoy CCHN services. Our analysis shows that our CCHN aligns well to industrial standardization activities and hence provides a viable approach to implementing future CRNs. We hope that our proposed design approach opens a new venue to future CRN research.

Index Terms-Cognitive radio networks, opportunistic spectrum access, multi-hop transmissions, spectrum auction, architectural design.

\section{INTRODUCTION}

$\mathbf{O}$ VER the last twenty years, we have witnessed tremendous success of mobile communications technology and dramatic increase of mobile data traffic. As reported in Cisco Visual Networking Index, by the end of 2015, mobile traffic has grown almost 400-million-fold over the past 15 years and will continuously increase by nearly 8 folds between 2015 and 2020 due to recent popularity of smart devices such as smart phones and various emerging applications, such as mobile health (mHealth), mobile online social networking, mobile gaming and mobile multimedia services [1]. For example, there are currently nearly 350 million smartphone, connected tablets and wearable devices being used in U.S. and generate more than 100,000 times the traffic supported in 2008 [2]. Such unprecedent proliferation of mobile data traffic will soon surpass the network capacity no matter how much spectrum allocated according to the Shannon's information capacity limits [3]. Hence, existing telecommunication systems will ultimately become congested and demand for more spectrum. Unfortunately, available spectrum for current telecommunication systems is very limited due to the fixed spectrum allocation policy. A recent study shows that, to support booming mobile data traffic, we will demand more than $350 \mathrm{MHz}$ additional licensed spectrum by 2019 [4]. In view of such a huge demand in extra spectrum resources, the Federal Communications Commission (FCC) recently opened up millimeter wave bands for $5 \mathrm{G}$ systems and relaxed the stringent spectrum policy in low frequency bands [5]-[8]. Along with the FCC's spectrum policy work, U.S. National Science Foundation (NSF) announced an over $\$ 400$ million investment to support advanced wireless research and dynamic spectrum sharing is viewed as a promising research direction [2]. Dynamic spectrum sharing enables opportunities for dynamically sharing under-utilized licensed spectrum bands as long as such a spectrum usage does not significantly impact 
the services of the incumbent licensed users. The resulting cognitive radio technology is viewed as a promising solution to achieving high spectrum efficiency by utilizing effective dynamic spectrum sharing strategies. According to [9], cognitive radio is an intelligent wireless communication system aware of its operating environment and dynamically reconfigures its operating parameters to efficiently utilize available spectrum bands. When equipped with cognitive radios, unlicensed users, called secondary users (SUS), could actively sense unused licensed spectrum and conduct communications accordingly without affecting the normal operations of the licensed users of that spectrum band, called primary users (PUs). Ever since the concept of cognitive radios was introduced, extensive research efforts have been made to the enabling technologies for cognitive radios, such as channel occupancy modeling, spectrum sensing, spectrum decision and resource allocation [10].

Recent research progress of cognitive radios has been well summarized in a couple of survey papers. As mentioned previously, the basic operations of a cognitive radio address awareness of the environment, decision making and reconfiguration of operating parameters. The first operation for a cognitive radio is to endow a radio with the ability to be aware of its operating environment. In the current literature, this is usually achieved via spectrum sensing. Recent advances on spectrum sensing have been surveyed in [11] where Ali and Hamouda [11] review existing approaches in narrow-band sensing, wide-band sensing and cooperative sensing, and the latest advances in implementation and standardization. In [11], developing compressive sensing based approaches in the presence of practical imperfections is listed as an important future step to push forward cognitive radio technologies. The latest advances related to this topic are surveyed in [12] and [13]. In [12], existing research works related to the application of compressive sensing in cognitive radios are thoroughly reviewed. In this paper, Sharma et al. first provide an introduction to compressive sensing and, then, survey existing works related to wide-band sensing, signal parameter estimation, radio environment map construction, and so on. In [13], a holistic review of main imperfections, which is possible to be encountered by a cognitive radio system, and corresponding countermeasures are provided. In addition to spectrum sensing, the capability of decision making is another requisite which makes radios cognitive. One of the most important decisions which should be made by cognitive radios is how to access PUs' spectrum. There are generally three kinds of spectrum access paradigms, i.e., opportunistic spectrum access (interweave), concurrent spectrum access (underlay) and cooperative spectrum access (overlay). When opportunistic spectrum access is adopted, cognitive radios cannot access licensed spectrum if PUs' activities are detected. Concurrent spectrum access and cooperative spectrum access allow cognitive radios to simultaneously exploit licensed spectrum with PUs. Concurrent spectrum access requires that interference received by PUs be controlled, while cooperative spectrum access requires SUs to assist PUs' transmissions in exchange for spectrum access opportunities [14]. Once spectrum access paradigms are determined, cognitive radios will decide which spectrum to use and how to adjust operating parameters accordingly. These problems are thoroughly discussed in [14] and [15]. Particularly, related machine learning techniques are comprehensively reviewed in [16] and [17]. When combining with accurate spectrum occupancy models, aforementioned techniques could lead to more efficient cognitive radios. In view of this, various spectrum occupancy models obtained from measurement campaigns all over the world are surveyed in [18].

Clearly, the spectrum scarcity and the development of cognitive radio technologies will eventually lead to the wide application of cognitive radios and the so-called cognitive radio networks (CRNs). One of the most crucial parts of CRNs is how to develop efficient resource allocation schemes to allow the coexistence of multiple cognitive radios. Due to its importance, resource allocation in CRNs has been well investigated in the literature. The general descriptions of the various aspects of resource allocation in CRNs, including important criteria, typical requirements/parameters and common approaches, are provided in [19] and [20]. A comprehensive survey of resource allocation algorithms are presented in [21] where similarities and differences as well as strengths and weaknesses of these algorithms are investigated. In [22], existing technical solutions related to cognitive MAC (C-MAC) design are reviewed based on the concept of C-MAC cycle. In [23], auction-based approaches are surveyed as efficient resource allocation methods for wireless systems, including CRNs. Specific resource allocation problems related to CRNs with the concurrent spectrum access paradigm and the cooperative spectrum access paradigm are discussed in [24] and [25], respectively. In CRNs, particularly multi-hop CRNs, another important issue is how to identify the best route for data delivery. A survey and a taxonomy of up-to-date routing metrics for CRNs are presented in [26]. In parallel with resource allocation and routing, there is an increasing research interest on security issues in CRNs. Recent advances in security threats and corresponding countermeasures in CRNs are studied in [27] with an emphasis on the physical layer, while a specialized but comprehensive survey on the Byzantine attack and defense for cooperative spectrum sensing in CRNs is presented in [28]. Recently, the growing concern in energy consumption has led to fast development of green communication technologies and the emergence of green-energy-powered CRNs. In [29], the state-of-the-art progress on energy-efficient cognitive radios and green-energy-powered CRNs is reviewed.

Clearly, the success of CRNs will not only depend on various technical progress mentioned above but also rely on an effective and efficient network architecture. As pointed out in [30], the potentials of aforementioned cognitive radio technologies might not be fully exploited without an appropriate network architecture. Unfortunately, this problem is still not well addressed. Even if there are some network architectures proposed in [10] and [31], it is still assumed that all end devices must be equipped with cognitive radio capability. Without an appropriate network architecture, the corresponding CRNs might even not be able to work correctly [15], [32]-[37]. Although there are lots of surveys and tutorials on CRNs, the importance of network architecture 
TABLE I

LIST OF ABBREVIATIONS

\begin{tabular}{|c|c|}
\hline Abbreviation & Definition \\
\hline PU & Primary user \\
\hline SU & Secondary user \\
\hline CRN & Cognitive radio network \\
\hline CCC & Common control channel \\
\hline ICRN & Infrastructure-based cognitive radio network \\
\hline CRAN & Cognitive radio ad hoc network \\
\hline HCRN & Hybrid cognitive radio network \\
\hline CCHN & Cognitive capacity harvesting network \\
\hline SSP & Secondary service provider \\
\hline BS & Base station \\
\hline CR-router & Cognitive radio router \\
\hline
\end{tabular}

design for CRNs has unfortunately been overlooked. In this paper, we attempt to fill in this void by providing a tutorial on network architecture design for CRNs. In this tutorial, we systematically summarize the principles of CRN architecture design and employ a novel flexible network architecture, called a cognitive capacity harvesting network (CCHN), as an example to elaborate on how to design an effective network architecture for CRNs. Unlike existing proposals, we introduce a new network entity, called a secondary service provider $(S S P)$, and deploy cognitive radio capable routing entities, called cognitive radio routers (CR-routers), in order to provide services to SUs more efficiently. According to the subsequent discussions, the $\mathrm{CCHN}$ can efficiently exploit available harvested spectrum bands to serve SUs without imposing constraints on their communication devices, which aligns well to current industrial standardization activities. Then, we present how to refine the designed architecture with multiple potential research directions. Finally, we demonstrate the effectiveness of the designed CCHN architecture by examining how it could be employed as a solution to CRNs in various application scenarios. To facilitate the easy reading, Table I summarizes the important abbreviations and their definitions.

The rest of this tutorial is organized as follows. In the following section, we review the important but widely overlooked challenges in practical implementation of CRNs and discuss how these deficiencies will affect the implementation of CRNs, which motivates us with a couple of design principles. Then, in Section III, we present our CCHN architecture as an example to elaborate on CRN architecture design and explain how it is related to the obtained design principles, current industrial standardization activities, and the existing proposals for CRNs. In Section IV, we articulate what kinds of problems have to be considered to further refine the details of the designed architecture with multiple potential research directions, followed by several possible interesting application scenarios most suitable for CCHNs in Section V. Finally, conclusions are drawn in the last section.

\section{Design Principles For CRN ARChitectures}

We first provide a holistic overview on the important but widely overlooked challenges in practical implementation of CRNs, which renders us a better understanding of CRNs and enables us to figure out important features that a future CRN must have, and thus guides us to come up with the fundamental design principles for the future CRN architecture. In what follows, we will examine those challenges from five aspects, namely, hardware implementation, common control channel establishment, efficient resource utilization, spectrum auction, and statistical end-to-end quality of service provisioning.

\section{A. Hardware Implementation}

Most of existing works assume each device has cognitive radio capability. However, it is unlikely that all devices would have this capability in place. Light-weighted handheld devices only have limited resources, such as computational resources, battery power and storage, which prevents them from achieving the goal required for cognitive radios. As required by the FCC, SUs should not cause harmful interference or disruptions to the transmissions of license holders, i.e., PUs. Thus, SUs should keep monitoring/sensing the unused spectrum bands to ensure that the PUs' transmissions are not affected, which will consume significant amount of time, computational resources and energy [32], [38]-[41]. When PUs are detected to be active, SUs' devices need to immediately vacate its current occupied licensed spectrum bands and possibly switch to other unlicensed/licensed spectrum bands. As reported in [42] and [43], switching between different spectrum bands will involve frequent tuning of power amplifiers, which incurs high energy consumption. Frequent spectrum sensing and spectrum switching processes will drain battery power very fast, and hence light-weighted devices may soon run out of battery power, making devices useless. On the other hand, relatively small sizes of light-weighted devices make hardware design challenging. In cognitive radios, we often need a reconfigurable antenna for communications and processing and another dedicated antenna for spectrum sensing/monitoring [44]. When implementing these antennas on small devices, we need to deal with coexistence issues, such as crosstalk, so that the performance of cognitive radios will not be significantly impacted. Moreover, when light-weighted devices utilize multiple spectrum bands simultaneously as suggested in various works, their design will be even more complicated since the devices should accommodate multiple transceivers/RF chains. Due to complexity in hardware design, endowing light-weighted devices with cognitive radio capability is not an easy task at the very least in the near future. Even if aforementioned challenges could be overcome, significant amount of time and effort should be devoted to hardware design and signal processing, which will inevitably increase the cost and complexity on the user side. It would be ideal that future CRNs could be designed to be flexible enough so that devices without cognitive radio capability could still benefit from cognitive radio technology. It would be also better to shift hardware design complexity from the user side to the network side as commonly done for many prolific applications. ${ }^{1}$ More importantly, the requirement for backward compatibility also

\footnotetext{
${ }^{1}$ It should be noted that our argument focuses on the network access part and thus does not contradict with the end-to-end argument in computer systems design [45], [46].
} 
calls for such a flexible CRN architecture to support services for existing devices without cognitive radio capability.

\section{B. Common Control Channel Establishment}

To efficiently utilize the harvested spectrum bands, we must ensure two parties between a link stay on the same channel and different links will not conflict/interfere with each other. In the current literature, this is usually achieved through a common control channel (CCC) where negotiation and information exchanges are carried out [47]-[50]. Unfortunately, it is still unclear which bands the CCC should be allocated. The motivation for SUs to access unused licensed bands is the lack of available spectrum bands to support current services. Thus, without proper provisioning of control channels, these SUs might not have enough reliable spectrum resource even for the CCC. Some works suggest using harvested bands to set up the CCC [48]. Due to spatial variation in PUs' activities, SUs might have different views of spectrum availability. In this case, how can we construct a CCC among SUs considering the discrepancy in spectrum availability? A rendezvous process could achieve this goal, but it will take relatively long time for SUs to converge on the same spectrum [51], [52]. In [48], it was proposed to employ a centralized approach to establishing the $\mathrm{CCC}$, which is more time efficient. Considering spatial variation of spectrum availability, centralized approach relies on SUs themselves to report their spectrum availability information. How and through which bands SUs could submit these information to the central controller is still unclear. Furthermore, employing harvested spectrum bands for control signaling will need to consider reliability issues. One basic premise of current cognitive radio technology is that SUs should vacate the spectrum immediately once PUs return. Due to the unexpected return of PUs, control signaling transmissions might be interrupted at any time, which might cause severe problems for normal network operations. Thus, to take advantage of cognitive radios, CRNs should be able to reserve reliable spectrum bands for control channels, yet this issue has not been well addressed in the current literature.

\section{Resource Utilization}

Even if hardware implementation and common control channel establishments have been addressed properly, spectrum resources may not be efficiently utilized if only based on current network architecture of CRNs [53]-[56]. One of the widely adopted CRN architectures is the infrastructurebased CRN (ICRN) where SUs connect with BSs/APs via single-hop connections. Such an architecture may lead to inefficient utilization of network spectrum resources. On the one hand, single-hop relaying schemes might result in long distance transmissions from SUs to BSs/APs and thus require relatively high transmission power, which reduces the number of available harvested bands to local SUs and hence lowers the utilization of locally available spectrum bands. On the other hand, single-hop transmissions will dramatically limit frequency reuse due to high transmit power and increase SUs' energy consumption. These issues could be resolved by incorporating multi-hop transmissions into ICRNs [57], [58].
However, current research works on multi-hop CRNs mainly focus on cognitive radio ad hoc networks (CRANs), which does not rely on fixed infrastructure and data is forwarded among SUs via multi-hop transmissions. CRANs encounter many challenges in practice [56]. Since data delivery in CRANs relies on per-user based routing, in order to obtain optimal routes, each flow initiator, the source, should acquire full network information, such as network topology, traffic information, spectrum availability, and residual battery energy, which may generate too much control overhead. Currently, how and on which bands these kinds of information is exchanged are still unclear. Moreover, in per-user based routing, even though SUs could make routing decisions, these routes might not be feasible due to the conflicts among them when considering spectrum availability. Even if the selected routes are feasible, it is difficult to allocate enough resources among different links and schedule their transmissions to build up these routes. Even if resource allocation and link scheduling could be resolved, how to disseminate the decisions is still challenging due to the lack of spectrum resources. More importantly, in case of link failure, how to notify the affected SUs is not an easy task. If the affected SUs are not timely informed, the allocated network resources might be wasted and the QoS of the affected sessions will inevitably be degraded. Thus, existing CRNs fail to achieve efficient resource utilization. From previous discussions, we conclude that an effective CRN should smoothly integrate ICRNs with CRANs and is capable of exploiting the benefits of both ICRNs and CRANs.

\section{Spectrum Auction}

In the current study of CRNs, spectrum auction is viewed as an efficient economic-based approach to dynamic spectrum access (DSA) [59]. Although many interesting approaches have been proposed to enable spectrum auction, it is difficult to implement them in practice. Most existing works on spectrum auction are per-user based scheme where SUs need to select spectrum bands to bid and submit bids all by themselves [60]-[63]. As SUs do not subscribe services from primary networks, how could they get the information on spectrum availability and submit their bids to the spectrum market? These could be handled by the auctioneer, but it is still unclear who the auctioneer is in current envisioned CRNs. Due to possible conflicts of interest, both spectrum sellers (i.e., PUs) and buyers (i.e., SUs) are not suitable to act as the auctioneer for many spectrum auction schemes proposed in [64] and [65]. Even if this is not the case, how and through which bands could the spectrum seller and buyers communicate with each other when one of them is served as the auctioneer? If the auctioneer is a third-party, how could SUs submit their bids to this third-party [66]? Even if all aforementioned issues have been addressed satisfactorily, current per-user based scheme might still be impractical because SUs might even not know what spectrum is due to the lack of expertise in telecommunications.

Besides, it is also difficult for the available spectrum resources to be efficiently utilized with the current peruser based spectrum auction scheme. First, existing spectrum 
auction schemes mainly focus on the auction schematic design and neglect corresponding implementation issues such as communication and information enabling services [61]. On the one hand, it is unclear whom the winning SUs communicate with and how to deliver the spectrum information to the other parties. On the other hand, it is unclear what kinds of services will be carried over the purchased spectrum bands. Second, due to limited network information, SUs might experience difficulties in determining the optimal spectrum bands to bid for, particularly when multi-hop transmissions are involved [67]. In this case, several mutually-interfered SUs might bid for the same spectrum and leave some other spectrum bands unused, which makes the auction process inefficient. Besides, when multi-hop CRNs adopt the per-user spectrum auction, a source SU could only bid for the first-hop, but could not bid spectrum for the relaying nodes unless a holistic end-to-end (session-based) spectrum auction scheme is used [67]-[69]. Apart from these issues, current per-user based schemes will experience difficulties in FCC rule enforcement and bill collection, particularly when online auctions are on the scale of minutes with costs on the scale of sub dollars. Clearly, none of these issues could be resolved without the support with some reliable network service provisioning. Since most current CRNs fail to fulfill this task, we need to design a novel CRN to provide network-wide support for spectrum auction.

\section{E. Statistical Quality of Service Provisioning}

In wireless networks, end-to-end (e2e) quality-of-service (QoS) provisioning is challenging but important [70]-[75]. Thus, in spite of its difficulty, there are still many research works done in the current literature attempting to address QoS issues in mobile ad hoc networks (MANETs) or wireless sensor networks (WSNs) [70], [71], [76]-[78]. Unfortunately, most existing works on CRNs are still focusing on one-hop transmissions without considering e2e QoS. Although quite a few papers have formulated flow optimization problems to address e2e QoS, many of them assume that PUs will not return during the optimization interval, leading to the flow optimization problems similar to those for multi-channel multi-hop networks, which is obviously inappropriate for practical CRNs. Unlike MANETs or WSNs, service delivery in CRNs is done by using others' spectrum resources opportunistically, which makes QoS provisioning in CRNs much more challenging. To guarantee the e2e QoS over a multihop connection in CRNs, we have to manage the spectrum resource in each hop in terms of spectrum harvesting, selection of appropriate spectrum bands, and maintenance of links. Obviously, if only harvested dynamic spectrum bands have to be used for CRN operations, an SU itself alone will not be able to address e2e multi-hop connection services, particularly when spectrum auction is used for acquisition of spectrum bands to sustain multi-hop transmissions. First of all, neighbor discovery has to be used to find potential relay nodes, but it is spectrum-dependent: over which band communications can be conducted. Given the uncertainty of harvested spectrum bands, how to find neighboring nodes become uncertain.
Even if we have discovered all neighbors, it may be difficult to select the appropriate band for that particular link without control channel provisioning because the transmitter and the receiver of a link may have different views of a band, and hence it may take intensive signaling exchanges and time to converge on the right harvested band for both the transmitter and the receiver, even if a reliable channel is available. Third, deterministic QoS guarantee seems not appropriate over CRNs when relying on uncertain harvested spectrum bands, and we have to seek statistical QoS guarantee for e2e service provisioning. Depending on application scenarios, statistical QoS provisioning can be handled differently. When the e2e paths have been fixed as considered in [79], the statistical QoS is provided by ensuring the traffic requirements on all the links along the considered path are simultaneously satisfied with certain probability. Under the assumption that spectrum availability in different links is independent, such a probabilistic constraint can be reformulated as separate probabilistic constraints for each link, which facilitates the solution finding. When spectrum availability of different links is not independent, we can find approximations/bounds of the constraints or try to decompose it into multiple simpler constraints with, for example, Bonferroni's inequality [80]. When the e2e paths are not determined beforehand as considered in [69], the statistical QoS can be provided by statistically quantifying concerned metrics, such as throughput and delay, of each link and performing network routing accordingly to select paths to meet the QoS requirement. In this case, a new set of novel stochastic optimization problems may have to be formulated to address e2e QoS issues. However, as mentioned earlier, without network-wide information, it is hard to address even e2e statistical QoS guarantee. As a result, e2e QoS provisioning in CRNs is impossible without effective support of the network-wide supervision. As far as we know, our recent works [67], [68] are among the few which tackle statistical QoS in CRNs under uncertain spectrum availability.

In summary, all above challenges imply that a successful $\mathrm{CRN}$ should be able to benefit non-CR devices (devices without cognitive radio capability), reserve reliable spectrum bands for control signaling, exploit the benefits of both CRANs and ICRNs, provide network-wide support for spectrum auction, and enable statistical QoS provisioning. Since existing CRNs are not flexible enough to meet all these challenges, we will present a novel flexible network architecture, namely, the cognitive capacity harvesting network (CCHN) architecture, to elaborate on how a CRN architecture could be designed to satisfy all those design principles. The central idea is to take a holistic approach from the end-to-end perspective to effectively and efficiently managing uncertain harvested spectrum resources to support randomly varying users' service demands because users are ultimately concerned with their end-to-end service quality, not how this is done. This cannot be achieved without network-wide perspectives of both service demands and resource availability as random variations in service demands and available resources can only be effectively managed with (at least partially) collective view on demands and resources. The proposed CCHN architecture reflects this 


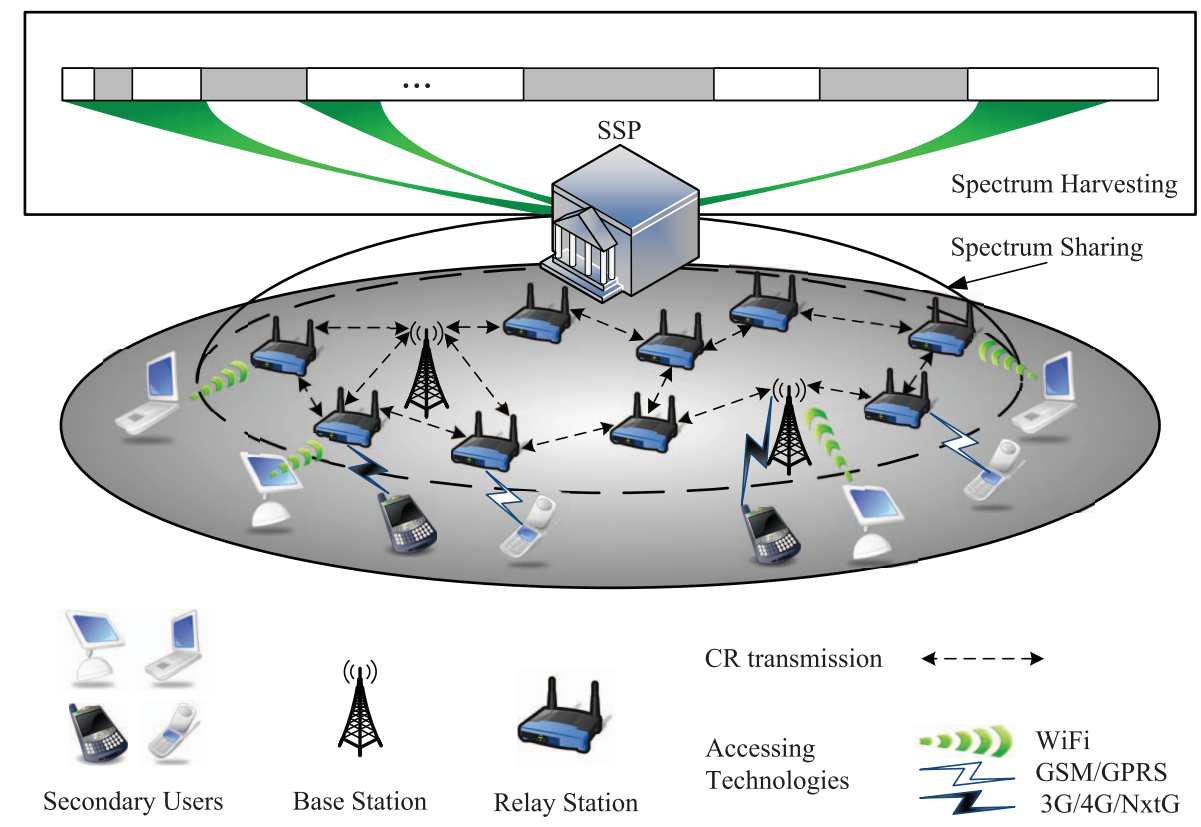

Fig. 1. The Cognitive Capacity Harvesting Network Architecture (CCHN) [69]. CR-routers are represented as relay stations.

design philosophy. In what follows, we will discuss this architecture in detail.

\section{CCHNs: COGNITIVE CAPACITY HARVESTING NETWORKS}

In this section, we first introduce our network architecture and basic network components, such as the secondary service provider (SSP), BSs and CR-routers. We then elaborate on how these network entities work together to efficiently support various kinds of SUs and their heterogenous traffic demands. We also provide detailed discussions on how the aforementioned design principles in the last section can be satisfied and reflected via the architecture design. Specifically, we incorporate the idea of control/user data $(\mathrm{C} / \mathrm{U})$ plane decoupling into our design in order to make the network operations more controllable and robust against random variations in both service demands and spectrum resource availability [81]. Moreover, we will discuss how SUs' data traffic is delivered and how available network resources, such as spectrum bands, could be efficiently exploited to support SUs' traffic with diverse volumes and QoS constraints. Furthermore, we provide a detailed description to show that spectrum auction can be easily carried out under the proposed CCHN architecture. Finally, we carefully study how the design architecture is related to industrial standardization activities as well as the existing architectures of CRNs.

\section{A. The Network Architecture}

The proposed CCHN is shown in Fig. 1 as first proposed as earlier as in 2011 [67], [69], [82], [83]. A unique feature of the $\mathrm{CCHN}$ is the introduction of a secondary service provider (SSP) which owns some basic licensed spectrum bands to provide basic reliable communication services such as common control signaling. The SSP can be an independent wireless service provider which is willing to provide new kinds of services to mobile users or existing wireless network operator which intends to enhance its existing services. For example, an SSP could be a cellular service provider (CSP) if the cellular operator experiences spectrum shortage or traffic congestion. We assume that the SSP has already deployed or is willing to deploy necessary partial infrastructure, such as BSs, CR-routers and spectrum sensing nodes, to serve its users. The BSs are used for fundamental coverage just as what has been done in cellular networks. The CR-routers form a wireless mesh backhaul, a cognitive radio mesh, to assist BSs in service delivery and could relay SUs' traffic in a multi-hop fashion. ${ }^{2}$ In the $\mathrm{CCHN}$, all BSs and $\mathrm{CR}$-routers have cognitive radio capability and are capable of utilizing both basic and harvested bands for communications. Under this architecture, SUs could be served by either BSs or CR-routers depending on their locations. For non-CR SUs, BSs and CR-routers could tune to, for example, the basic bands to deliver mobile services. If SUs carry cognitive radios, they could communicate with BSs and CR-routers over both the basic and harvested bands. In the following, we will give a detailed description of the network entities and how they work together to offer services to SUs.

As stated above, an SSP is a wireless service provider which has its own basic licensed spectrum bands and partial infrastructure, such as the CSP. An SSP can be the owner as well as the operator of the CCHN and is responsible for its normal operations and maintenance. The SSP is in charge of resource allocation and coordination of CR-routers to provide services to SUs in its coverage area. For this purpose, the SSP needs to exchange control information with CR-routers

\footnotetext{
${ }^{2}$ In this paper, SUs refer to those users who have subscribed the SSP's service.
} 
TABLE II

IMPORTANT CONCEPTS IN THE CCHN

\begin{tabular}{|c|c|}
\hline Concept & Definition \\
\hline SSP & An service provider that coordinates spectrum harvesting and optimization within the CCHN \\
\hline PU & The incumbent devices that possess the licenses for specific spectrum bands \\
\hline BS & $\begin{array}{c}\text { An infrastructure node provides the fundamental support of coverage services, allows the SSP to gain } \\
\text { backbone network services and serves as an agent of the SSP for control message exchange }\end{array}$ \\
\hline CR-router & $\begin{array}{r}\text { A wireless router/fixed relay station equipped with multiple CR interfaces, which can be tuned to various } \\
\text { available frequency bands for communications }\end{array}$ \\
\hline Cognitive radio mesh & $\begin{array}{r}\text { A wireless mesh backhaul which could employ both harvested bands and basic bands } \\
\text { to assist BSs in service delivery via multi-hop transmissions }\end{array}$ \\
\hline Basic bands & Spectrum bands licensed to the SSP \\
\hline
\end{tabular}

through BSs. Thus, the SSP will reserve a certain number of basic bands for the common control signaling between BSs and CR-routers. Besides, to enable user access, it will also allocate a certain number of resources, including a few basic bands, to BSs/CR-routers for SUs' data delivery and user access related control signaling. The SSP will allocate the remaining basic bands, together with harvested licensed bands, to the cognitive radio mesh of CR-routers for data delivery. It could employ CR-routers to proactively collect intelligence, e.g., spectrum availability and the distribution of network traffic, from the network. For example, with the spectrum availability information collected by CR-routers and spectrum sensing nodes, the SSP could construct a fine-grained spectrum map over its service area. When running out of licensed spectrum bands, the SSP could immediately identify unused harvested licensed bands to use. With the collected information, it can make centralized scheduling and resource allocation, and send the scheduling decisions to CR-routers to coordinate their operations. Furthermore, the SSP could also purchase unused licensed spectrum bands directly from spectrum markets when necessary.

The BSs can be deployed by the SSP to provide basic coverage services or can be leased from existing cellular operators (e.g., the SSP could lease certain amount of bandwidth from a cellular operator to save initial deployment cost or just lease the use of BSs but with its own transceivers and spectrum bands). BSs are interconnected with wired connections via either the Internet or other data networks or Public Switched Telephone Networks (PSTNs), which allows the SSP to gain connections to the backbone network. On the one hand, BSs will serve as a data aggregation point or a gateway for CR-routers. On the other hand, BSs act as agents for SSP to exchange control signaling information with CR-routers and SUs. Similar to its counterpart in cellular networks, the BSs in the CCHN could support control signaling as well as data communications. Each BS has its own coverage area, i.e., a cell, and SUs could connect with BSs in their proximity, particularly when they have urgent data to transmit. BSs are equipped with multiple radios and air interfaces, such as the basic band interface, the cognitive radio interface and the WiFi interface. Depending on other parties' available interfaces, BSs will select proper interfaces to communicate.

A CR-router could be either a wireless router installed at certain locations (e.g., lampposts, rooftops, or trees) or integrated in a mobile node. Similar to BSs, CR-routers are equipped with multiple radio interfaces, such as basic band interfaces and cognitive radio interfaces. Thus, CR-routers could communicate over both the basic bands and the harvested licensed or unlicensed bands. CR-routers could work collaboratively with mobile sensing nodes to sense the licensed/unlicensed spectrum bands and forward the results to the SSP to build up a fine-grained spectrum map over the SSP's service area. The CR-routers could support basic control signaling via reliable basic bands, including system information broadcasting, handover signaling and signaling for resource allocation, such that SUs could directly connect to CR-routers [84]. Each SU selects a nearby CR-router/BS to connect based on certain metrics, such as the maximum Reference Signal Receiving Power (RSRP) and the maximum receiving signal-to-interference-andnoise-ratio (SINR) [85], [86]. CR-routers are responsible for providing their associated SUs with backhaul connection to backbone networks and coordinate the transmissions of these SUs. CR-routers collect the traffic requests of their associated SUs and submit the aggregated requests to the SSP for routing, scheduling and resource allocation. Once the scheduling decisions have been made, CR-routers work collectively to deliver data traffic accordingly. Based on the resources allocated by the SSP (e.g., spectrum bands, time periods), CR-routers will perform local resource management to guarantee SUs' experience.

In summary, BSs and CR-routers collaboratively work together to manage the resources (licensed or unlicensed) to handle the traffic in the coverage area. The CR-routers form the cognitive radio mesh to transport data from BSs closer to the end users (SUs) so that spectrum can be more efficiently utilized with potentially higher frequency reuse. For clarity, the important concepts involved in the CCHN are summarized in Table II, and the cognitive cycle and typical cognitive radio activities involved in the CCHN are shown in Fig. 2. Up to now, we explain why the designed architecture is characterized by "capacity harvesting". By "capacity harvesting", we mean that the proposed network architecture is able to identify and utilize unused spectrum resources which can provide the network with extra capacity. "Capacity harvesting" is a similar concept to energy harvesting since both of them are related to the process of acquiring extra resources from surrounding environment [29]. Unlike energy harvesting, "Capacity harvesting" includes not only the process of identifying available spectrum bands but also the process of utilizing these spectrum bands, which is the reason why we choose "capacity harvesting" instead of "spectrum harvesting". In fact, the proposed network architecture can also identify other capacity such as 


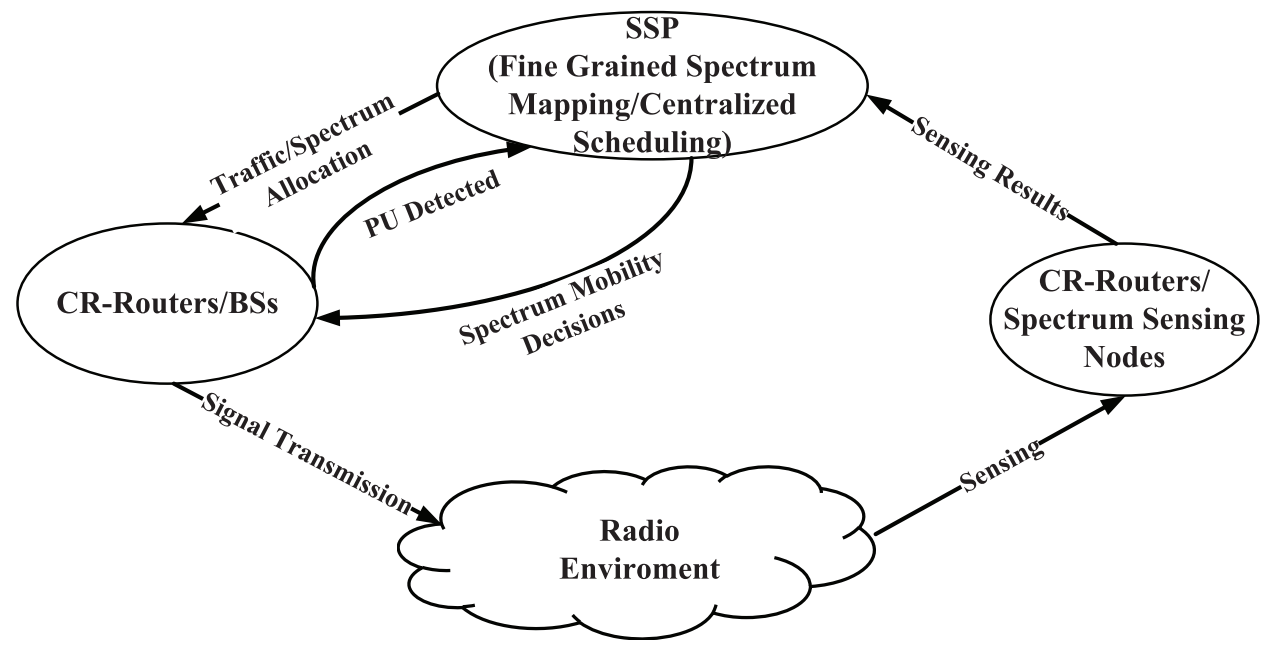

Fig. 2. The cognitive cycle and typical operations in the CCHN.

energy saving facilities, high speed connection points, etc., which will be investigated elsewhere to avoid confusion in this paper.

\section{B. C/U Plane Decoupling}

Control signaling plays a very important role in managing overall communications services and must be supported by reliable channels for fast connection establishment and for quick opportunistic resource allocation. Due to the uncertainty of harvested spectrum caused by random returns of PUs, harvested spectrum will be better managed by reliable control signaling protocols. Since, in our CCHN, we require that the SSP have its own basic bands to support control signaling while harvested spectrum support data services, we naturally have control and user data planes $(\mathrm{C} / \mathrm{U})$ decoupled. This is also why we introduce the basic bands to enable $\mathrm{C} / \mathrm{U}$ decoupled protocols to more effectively manage the use of harvested spectrum.

Besides, different SUs might have very diverse moving speeds, such as the smartphones of pedestrians and moving vehicles. It is always better to handle connections based on mobility. Usually, it would be better to connect fast SUs to BSs for services to lower handoff rate while connecting slow SUs to CR-routers as commonly done in cellular systems, particularly in $5 \mathrm{G}$ cellular systems. When SUs move in high-speed, if we purely rely on traditional coupled signaling protocols to manage connections, they may be connected to CR-routers for their services, and hence there will be more inter-CR-router handoffs. Too frequent handoffs will cause not only too much control signaling traffic but also poor user experience due to potential disconnections caused by failed handoffs [87]. To reduce control signaling overhead and enhance user experience during handoff, in our proposed $\mathrm{CCHN}$, we can use $\mathrm{C} / \mathrm{U}$ decoupled scheme to proactively manage the connections as we mentioned before and also as we have done for high-speed railway systems [81]. For high-speed SUs, e.g., User 2 in Fig. 3, the control plane (C-plane) traffic is directly managed by the BS, while the data plane (U-plane) traffic is handled by

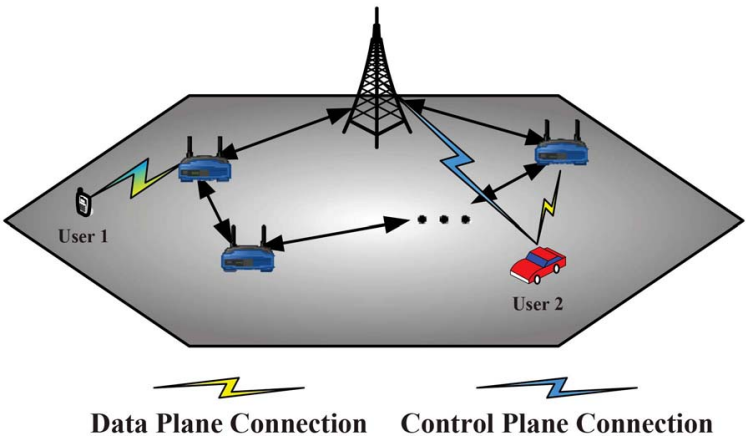

Fig. 3. $\mathrm{C} / \mathrm{U}$ Plane Decoupling.

either the BS directly or CR-routers, but controlled by the BS. Namely, the C-plane and U-plane traffic are managed by separated nodes. If an SU's data service is via a BS, C-plane and U-plane will conduct handoff simultaneously. If a SU's data service is via CR-routers, the connected BS will manage the C-plane and proactively plan the handoff between CR-routers to simplify the handoff process. We can also utilize the bicasting strategy proposed in [81] to enable the current serving CR-router and target CR-router to provide data services during the handoff area. Since the serving BS has more information about the spectrum resource availability and mobility of the SU, we can address handoff more efficiently while maintaining seamless services statistically. For low speed SUs, e.g., User 1 in Fig. 3, we still let both the control signaling and the data traffic to be handled by CR-routers. In such a way, basic bands for control signaling could be reused due to the short range connections between SUs and CR-routers. By aggregating the traffic from SUs with low mobility, BSs will handle fewer connections and thus leave more room to manage more high-speed SUs. As a remark, the proposed control signaling protocol is aligned well to the design methodology of the emerging software-defined networks (SDNs). The C/U decoupling also bears similarity to the DUCHA protocol we proposed for IEEE 802.11 networks which has been shown to significantly improve network performance [88]. 


\section{Data Delivery for SUs}

Data transmission in the proposed CCHN is highly flexible as a cognitive radio mesh is added between BSs and SUs. According to the types of services, the data traffic of SUs could be delivered through either long-distance direct connections (i.e., the SU $\leftrightarrow \mathrm{BS} \leftrightarrow$ the data network) or short-range multi-hop transmissions (i.e., the SU $\leftrightarrow$ CR-router $\leftrightarrow \cdots \leftrightarrow$ $\mathrm{BS} \leftrightarrow$ the data network). When requiring low-latency services, SUs will directly exchange their data with the nearby BSs. Otherwise, SUs interact with the data network via their associated CR-routers and the cognitive radio mesh where SUs' data are delivered through multi-hop transmissions. If the data are delivered between two SUs in the same service area, it could even be carried via the short-range multi-hop transmissions without going through BSs (i.e., the source SU $\leftrightarrow$ CR-router $\leftrightarrow \cdots \leftrightarrow$ CR-router $\leftrightarrow$ the destination SU).

For SUs connecting to CR-routers, they first submit their online data requests to their closest associated CR-routers at the beginning of each scheduling period. Then, CR-routers aggregate those requests and submit them to the SSP (i.e., the associated BS). Based on the collected information, the SSP formulates network optimization involving routing, link scheduling, and resource allocation by considering traffic demands, spectrum availability, and energy consumption. Once the optimization is carried out, the decision is sent to CR-routers through the C-plane. Accordingly, CR-routers work collectively and collaboratively to deliver SUs' data over the cognitive radio mesh through multi-hop transmissions. Meanwhile, CR-routers notify the admission/rejection of the transmission requests under their coverage. Once being admitted, SUs transmit their data to, or receive data from, $\mathrm{CR}$-routers via the allocated resources. For user devices without cognitive radio capability, their communications with BSs/CR-routers are conducted through the basic bands or the bands which they normally use for communication services. In other cases, according to the resource allocation decisions, SUs could communicate with BSs/CR-routers through either basic bands or harvested bands. Notice that the cognitive radio mesh is attempting to minimize the use of basic bands and transport data as close as possible to the end users. In this way, some data services, such as delay tolerant services (e.g., video clips), can be offloaded to harvested bands while saving basic bands for more important services such as delay sensitive services.

\section{Traffic Differentiation in Cognitive Radio Mesh}

In our $\mathrm{CCHN}$, the significant amount of data traffic are relayed through the cognitive radio mesh. Given limited available spectrum bands, how to efficiently deliver SUs' traffic is key to the success of the CCHN. On the one hand, SUs' data requests come with different sizes and QoS constraints. When listening to music, users may expect more timely delivery of service and the size of a music file could be quite small. While downloading files, users could tolerate certain amount of delay and the size of a file could be relatively large. To efficiently support SUs' data traffic, we should differentiate data with different characteristics. On the other hand, in the proposed CCHN, particularly in the cognitive radio mesh, there are two kinds of spectrum bands, i.e., the basic spectrum bands and the harvested spectrum bands. The basic spectrum bands are reliable as they are owned by the SSP. The harvested bands are not reliable as the SSP do not hold a license on those bands and thus do not have priority. The premise of dynamic spectrum sharing is that SUs should vacate the spectrum bands once PUs become active. Due to the unexpected return of PUs, the harvested spectrum bands are not reliable enough to support delay-sensitive data or data with stringent QoS constraints. Consequently, in the cognitive radio mesh, these kinds of data are carried over the reliable basic bands, while delay tolerant data should be transmitted over harvested bands. As most of data traffic observed in today's mobile traffic such as video clips are delay tolerant [89], differentiating traffic according to their timeliness or QoS requirements could save precious basic spectrum to support more sessions with strict delay or QoS constraints.

\section{E. Improved Spectrum Utilization}

It is commonly observed that spectrum utilization can be improved via centralized control and multi-hop transmissions. As a network operator, the SSP is in charge of the spectrum allocation for the CCHN. With the help of CR-routers, the SSP collects various kinds of information, such as traffic distribution and unused licensed spectrum bands available to the network for opportunistic access. Based on gathered information, the SSP supervises the operations of the cognitive radio mesh by performing centralized network optimization to determine routing, link scheduling and resource allocation. This centralized control facilitates the efficient management of available spectrum bands including those unreliable harvested spectrum bands. Meanwhile, instead of using long-distance direct transmissions with BSs, the cognitive radio mesh allows SUs' data to be delivered via short-distance multi-hop communications, which will significantly improve frequency reuse and hence spectrum utilization. For example, multi-hop transmissions reduce the first-/last-hop transmission range of SUs. Due to the reduced transmission range, the transmit power of SUs/CR-routers could be significantly lowered and frequency reuse for basic bands could be significantly improved through careful frequency planning. For illustrative purpose, let us consider the CCHN shown in Fig. 4 where frequencies $f_{1}, f_{2}$ and $f_{3}$ are employed in cell 1 , cell 2 , and cell 3 for communications, respectively. Clearly, If SUs directly communicate with BSs, $f_{1}, f_{2}$ and $f_{3}$ may be different in order to guarantee performance according to the traditional frequency planning, which significantly limits the available basic spectrum bands for each cell. In contrast, with low-power multi-hop transmissions via CRrouters in our CCHN, user 1 in cell 1 not only could use frequency $f_{1}$, but also could borrow $f_{2}$ and $f_{3}$ from neighboring cells to exchange its data with the nearby CR-router when the transmit power of the CR-router or user 1 is kept at certain level. Moreover, thanks to the short-range multi-hop transmissions, both SUs and CR-routers could employ a lower transmit power level and thus locally unused licensed spectrum bands could also be utilized more efficiently. As shown in Fig. 5, PU1, PU2 and PU3 are PUs. Assuming that PU1 is 


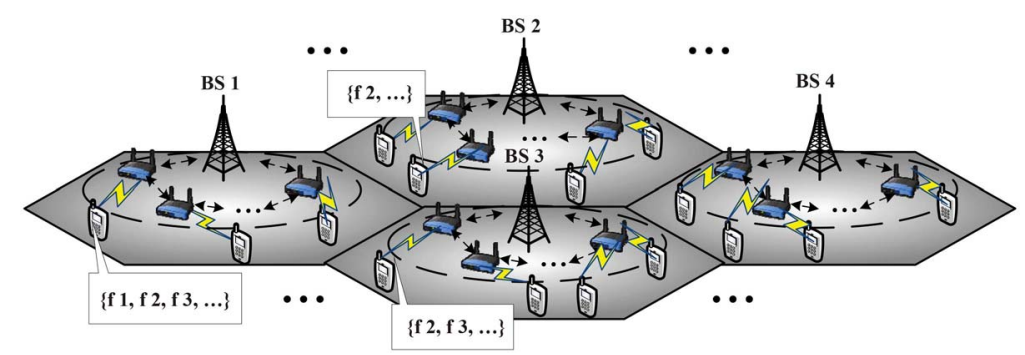

Fig. 4. The CCHN architecture when a cellular operator is the SSP.

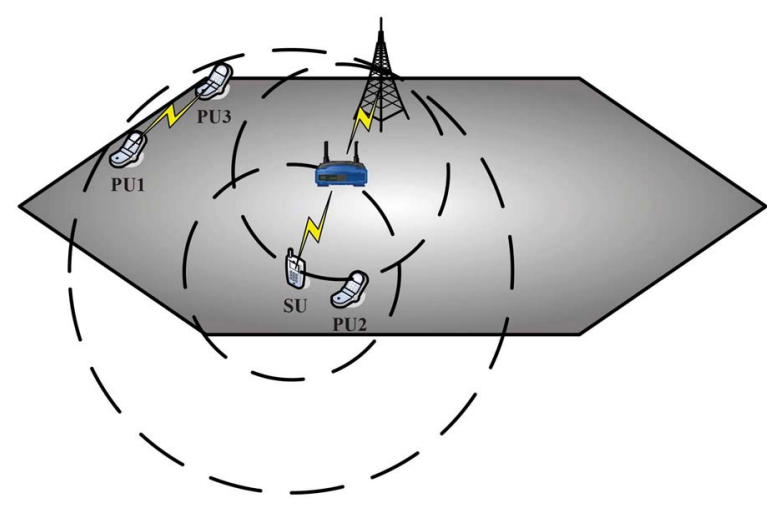

Fig. 5. SU transmissions over CR-Routers.

communicating with PU3 and PU2 is silent. The SU could not directly communicate with the BS via PUs' spectrum as PU1 and PU3 are within its interference range [67]. However, as shown in Fig. 5, due to reduced transmission power, the data of SU could be exchanged with the BS through the CR-router as a relay without interfering with the transmissions between PU1 and PU3.

\section{F. Spectrum Auction Support}

Unlike existing works, in the CCHN, it is the SSP which participates in the spectrum auction. The SSP aggregates SUs' data requests and their possible bids for services via its deployed CR-routers [90]. This process is carried out on the control channels or the channels allocated by the SSP. Upon receiving these data requests and their associated bids, the SSP makes centralized coordination to optimize the network performance or maximize its profit, depending on its revenue model. When the current available basic and harvested bands are not enough to support SUs' requests, it will determine how much spectrum is needed and then join the spectrum auction process to bid for the needed spectrum resources. Compared with per-user based spectrum auction schemes, the spectrum auction in the CCHN is feasible and practical. First, as an operator, the SSP has more bargaining power than individual SUs and owns more credibility to carry out spectrum auction. Second, the SSP possesses all necessary information, such as traffic distribution and spectrum availability, and thus is clear about which spectrum bands to bid for both in time and in space. In this way, the SSP could minimize the amount of purchased spectrum while guaranteeing certain level of satisfaction of SUs, the statistical QoS guarantee [91]. Third, since most of the participants in the spectrum market are SSPs rather than SUs, the number of involved parties in spectrum auction is dramatically reduced, which facilitates online auction and contract enforcement. Finally, since the SSP has its own OAM (Operations, administration and management) system, spectrum auction related operations can be treated as a part of its OAM, and hence bidding process can be implemented easily and does not need SUs to interact with the spectrum market place for any online spectrum auction operations.

\section{G. Advantages of the Proposed CCHN}

The architecture of the proposed CCHN is very flexible to implement future CRNs and could address almost all issues we have mentioned in Section II. First, we only require CR-routers and BSs rather than SUs to have cognitive radio capability. Compared with light-weighted SUs, CR-routers and BSs are less restricted in size and power, which makes the installation of cognitive radios easier. Since CR-routers and BSs are equipped with multiple interfaces, including the basic band interface and the cognitive radio interface, they are capable of communicating on both basic and harvested bands. SUs could communicate with $\mathrm{CR}$-routers/BSs via cognitive radios if they have the cognitive radio interface. If not, they could interact with CR-routers/BSs through basic bands or the bands which they normally use for communication services. In such a way, the hardware design complexity of CRNs is shifted from users' side to the network side and SUs could benefit from cognitive radio technology even if they do not have cognitive radio capability. On the one hand, even SUs communicate with CR-routers via basic bands, their data might be delivered over the harvested bands in the cognitive radio mesh. On the other hand, by shifting delay tolerant traffic to harvested bands, the cognitive radio mesh requires fewer basic bands and thus more basic bands could be saved to handle more QoS stringent services for SUs. Besides, since SUs do not have to conduct power hungry operations such as spectrum sensing, tremendous energy saving can be achieved on SUs, which will prolong SUs' battery life. Second, common control signaling is no longer a difficult issue since the SSP owns its basic bands and could allocate part of these reliable bands for control signaling. Third, the network resources could be efficiently utilized due to multi-hop transmissions and partially centralized (hybrid) control just as what has been done in cellular systems. As aforementioned, multi-hop transmissions in cognitive radio mesh not only facilitate more aggressive frequency reuse, but also enable better exploitation 
of locally unused harvested spectrum bands. In the CCHN, the SSP will employ CR-routers and spectrum sensing nodes to collect network intelligence, such as traffic distribution and spectrum information. Based on collected information, the SSP makes (partially) centralized network optimization and provides network-level supervision to support statistical QoS provisioning, which solves those problems faced by per-user based routing schemes in CRNs. For example, based on collected information, the SSP can statistically quantify the concerned metrics, such as throughput and delay, of each link and obtain optimal traffic and resource allocation strategies by formulating and solving cross-layer optimization problems accordingly with flow routing and link scheduling constraints [67], [90]. Then, the SSP will send corresponding decisions to BSs and CR-routers to coordinate their operations so that e2e QoS can be statistically guaranteed. Upon the return of PUs, the affected CR-routers will immediately stop transmitting and notify the SSP through the allocated control channels. Then, the SSP will find new paths for the affected sessions. Moreover, the flexibility and the cognitive capability of the CCHN make it possible for the SSP to efficiently exploit the big chunk of unlicensed millimeter wave bands [92]. Fourth, the SSP and its deployed infrastructure will provide efficient support for spectrum auction. In the $\mathrm{CCHN}$, SUs submit data service requests as well as their bids for data delivery services to the SSP via allocated channels. Upon receiving all these requests and bids, the SSP finds out how much spectrum resource is needed and submits bids to the auctioneer through, for example, wired connections. Since the SSP has all necessary information, it could easily identify the optimal spectrum bands to bid for. Due to the existence of the SSP, bill collection and FCC rule enforcement will become much easier. Fifth, with the SSP, the number of participants competing for spectrum access opportunities will be greatly reduced, which allows more intelligent access to PUs' spectrum bands. Since all the CR-routers and BSs are deployed by the SSP, the whole CCHN could be considered as a single party searching for spectrum access opportunities. Noticing PUs' traffic intensities are diverse over different spectrum bands and at different locations, the SSP could determine to access the optimal spectrum bands, such as those with light traffic or less variation in spectrum availability. For illustration purpose, let us consider cooperative spectrum sharing, where the SSP can coordinate its deployed BSs and CR-routers to help with PUs' transmissions in exchange for spectrum access opportunities, as an example [50], [93]. Since these BSs and CR-routers are owned by the SSP, when the SSP attempts to get spectrum access by cooperating with PUs, it could select a set of PUs to cooperate with to maximize the overall throughput of the CCHN. As the feasibility of cooperative spectrum sharing depends on PUs' and SSPs' willingness to cooperate, we can employ contract theory and two sided matching to design cooperative spectrum sharing schemes under the CCHN considering incomplete information, multiple PUs and multiple SSPs [94]-[96].

In summary, our CCHN design advocates the collective welfare of the whole SU community by introducing a new player, the SSP, to manage the spectrum harvesting to more effectively serve the whole SU community and by shifting the design complexity from user side to the network side. The collaborative design is aligned well to the design trend happening in $5 \mathrm{G}$ cellular systems and beyond. The effectiveness of the $\mathrm{CCHN}$ has been verified in various situations, the results are shown in some of our recent works [67]-[69].

\section{H. Relation to Industrial Standardization Activities}

The proposed CCHN could be seen as an ideal complement to existing industrial standardization activities. Currently, industrial standardization efforts mainly focus on operational specifications at the physical (PHY) layer and the medium access control (MAC) layer to implement efficient cognitive radio capability [97]-[102]. For example, the IEEE 1900.4 aims to define resource management building blocks in both network and user sides for coordinated decision making and efficient spectrum utilization, the IEEE 1900.6 aims to facilitate spectrum sensing by standardizing the information exchange between spectrum sensors and their client, such as spectrum databases and intelligent building blocks, and the standard ECMA-392 specifies a physical layer and a medium access control layer for personal/portable devices operating in TV white spaces [97], [103], [104]. While our CCHN aims to provide a flexible architecture for radio access networks where the potential of cognitive radios in boosting network capacity can be fully exploited. The $\mathrm{CCHN}$ aligns well to those industrial standards in how spectrum resources are identified and utilized, such as the adoption of spectrum usage maps and relatively centralized spectrum allocation. In the $\mathrm{CCHN}$, CR-routers and BSs have multiple interfaces which can be flexibly reconfigured by the SSP to efficiently utilize available spectrum resources for service delivery. In view of this, the CCHN also fits well to the application scenarios of cognitive radio systems listed by the ITU [105], [106]. In a word, various functional entities, such as spectrum usage maps, agile communication devices and functional architecture in CR-routers, of the CCHN can be implemented by following those industry standards, and the industry standards can be complemented by the CCHN to implement flexible and efficient CRNs. For convenience, we list major standardization body and their technical focuses in Table III.

\section{Current Proposals for CRN Architectures}

In the current literature, there are mainly three kinds of CRNs, i.e., CRANs, ICRNs and hybrid CRNs (HCRNs) [31], [50], [107], [108]. A CRAN does not rely on fixed infrastructure, and, in a CRAN, data is forwarded among SUs via multi-hop transmissions. Unlike traditional ad hoc networks, multi-hop transmissions in a CRAN are very challenging. Being distributed in nature, the end SUs should make routing decisions by themselves, which requires SUs to obtain various kinds of information such as network topology, traffic information and spectrum availability. Unfortunately, due to spatial and temporal variations in spectrum availability, information collection in a CRAN is very difficult and control signaling overheads are very high [31]. With limited information, routing decisions of SUs are inevitably subject to 
TABLE III

STANDARdization Bodies AND THEIR TeChNicAl FocUses

\begin{tabular}{|c|c|c|}
\hline \multicolumn{2}{|c|}{ Standardization Body } & Technical Focus \\
\hline \multirow[b]{2}{*}{ IEEE } & IEEE 802 Group & 802.22 standard for wireless RANs operating in TV white space \\
\hline & IEEE SCC 41 & $\begin{array}{l}1900 \text { standards for next generation radio } \\
\text { access networks and advanced spectrum management }\end{array}$ \\
\hline \multicolumn{2}{|c|}{ ECMA } & ECMA-392 PHY and MAC for operation in TV white space \\
\hline \multirow{4}{*}{ ETSI RRS TC } & WG1 & System aspects and cognitive functionalities \\
\hline & WG2 & Radio Equipment architecture \\
\hline & WG3 & Security, certification and declaration of conformity \\
\hline & WG4 & Civil security and inter domain synergies \\
\hline \multirow{5}{*}{ ITU } & WP 1A & Spectrum engineering techniques \\
\hline & WP 1B & Spectrum management methodologies and economic strategies \\
\hline & WP 1C & Spectrum monitoring \\
\hline & WP $5 \mathrm{~A}$ & $\begin{array}{l}\text { Land mobile service above } 30 \mathrm{MHz}^{*} \text { (excluding IMT); } \\
\text { wireless access in the fixed service; } \\
\text { amateur and amateur-satellite services }\end{array}$ \\
\hline & WP 5D & IMT Systems \\
\hline
\end{tabular}

transmission contentions as well as interference. Moreover, as the growing traffic demands are mainly targeted at Internet services, current end-to-end services promised by a CRAN might not be truly interesting in practice. In contrast, the CCHN architecture allows SUs to obtain data services by simply accessing to neighboring BSs/CR-routers. SUs only need to subscribe the SSP's services and submit their data requests to neighboring BSs/CR-routers. The SSP will collect SUs' data requests and spectrum availability information with the help of CR-routers. With collected information, the SSP will provide network-level supervision to BSs and CRrouters via its basic bands in order to deliver data services to SUs. Namely, the CCHN architecture alleviates SUs from the burden of information collection and decision making. In addition to CRANs, several existing works advocate ICRNs in which base stations (BSs) or access points (APs) serve as the anchoring points and provide SUs with backhaul connections to the Internet [50], [107]. In an ICRN, BSs/APs are equipped with cognitive radios and SUs could directly communicate with BSs/APs on unused licensed spectrum bands via single-hop transmissions, which dramatically limits frequency reuse and increases SUs' energy consumption. Additionally, current ICRNs encounter many design challenges in practical implementations. The basic premise in ICRNs is that BSs/APs are able to collect all necessary information and disseminate resource allocation decisions back to SUs [48], [50]. Unfortunately, information collection and dissemination in a CRN are also very challenging. SUs' demands for unused licensed spectrum bands imply that their allocated spectrum bands are not enough to support current traffic requests and they might not have extra spectrum bands to submit necessary information to BSs/APs. Without this information, it is difficult for BSs/APs to make efficient coordination and achieve efficient resource utilization. Even if coordination decisions have been made, SUs and BSs/APs might have different views of spectrum availability, and BSs/APs will encounter difficulties in issuing coordination decisions and establishing communication links. Unlike ICRNs, in the CCHN, the SSP employs dedicated basic bands to supervise the operation of the cognitive radio mesh where data traffic is delivered between SUs and BSs via multi-hop transmissions. In such a way, all aforementioned issues can be addressed under the CCHN architecture.
When compared with CRANs and ICRNs, HCRNs might be the architecture which is most similar to the CCHN, since, in both architectures, SUs' data are delivered to BSs via multi-hop transmissions. However, it should be noted that the CCHN architecture is substantially different from HCRNs. An HCRN consists of SUs with CR capable communication devices and BSs operating under different network access technologies [109]. SUs can access BSs, which might belongs to different service providers, either directly or through multi-hop transmissions with the help of other SUs. Namely, HCRNs are generally combinations of CRANs and ICRNs. Unlike HCRNs, our CCHN design advocates the collective welfare of the whole SU community by introducing a secondary service provider (SSP) with its own licensed spectrum bands, i.e., basic bands, to manage the spectrum harvesting and the cognitive radio mesh to more effectively serve the whole SU community. Different from HCRNs, when designing the CCHN, we do not impose specific requirements on SUs' communication devices so that SUs can enjoy the benefits of cognitive radio technology even without cognitive radio capability. To achieve this goal, CR-routers with different kinds of interfaces are introduced to facilitate SUs' network access. Unlike HCRNs where SUs' data is relayed to BSs with the help of other SUs, CR-routers in the CCHN form a cognitive radio mesh to facilitate data exchange between BSs and SUs. In contrast to HCRNs, all BSs and CR-routers in the CCHN belong to the SSP who can optimally exploit available network resources, such as harvested spectrum bands, to serve SUs. Moreover, different from HCRNs, the CCHN adopts the idea of using reliable basic bands to supervise the utilization of unreliable harvested bands in the cognitive radio mesh, which enables efficient resource utilization. Thus, the CCHN is a novel architecture for CRNs and is designed according to the principles summarized in Section II rather than a simple combination of the CRAN and the ICRN.

\section{Potential Research Directions}

In Section III, we elaborate, in general, on how a flexible and efficient architecture for CRNs could be designed based on the principles provided in Section II. To fully exploit the nice features of the designed CCHN, we need to refine the 
details of the design. In what follows, we will elaborate on what kinds of problems have to be considered to further refine the details of the designed architecture with several possible research directions.

\section{A. CR-Router Design}

A key component in the CCHN is the CR-routers which are responsible for gathering spectrum statistics, collecting SUs' traffic information, conducting data transmissions and receptions, and managing spectrum allocation in their charging regions. To fulfill all prescribed functions, besides routing capability, CR-routers should meet several requirements. First, each CR-router should be equipped with multiple radios such that it could exploit multiple spectrum bands simultaneously. Second, in case of the unexpected return of PUs, CR-routers should be able to monitor the harvested bands even if they are currently utilizing these bands. This requires CR-routers to be capable of handling self-interference. Third, each CRrouter must have multiple interfaces such that non-cognitive SUs can benefit from CRNs. Fourth, as required by the CCHN, each CR-router has to exchange control messages with SUs, BSs and other CR-routers. Considering possible deployment scenarios, say, lampposts, the size and power consumption of CR-routers should be kept as small as possible. The integration of all these required functions on a CR-router with relatively limited size and power consumption is not easy and significant research efforts are needed.

\section{B. CR-Router Placement}

The CCHN relies on CR-routers to deliver services, which implies the deployment of CR-routers will significantly affect the performance of the CCHN. To harness the benefit of the CCHN, CR-routers should provide a full coverage in the service area of the SSP. To achieve full coverage, the locations of CR-routers matter. When CR-routers are sparsely deployed, they might employ higher transmit power to provide coverage and thus more energy will be wasted on broadcasting access related information, such as CR-router identification and synchronization signaling. At the same time, the longer the distance between CR-routers and SUs, the more transmit power SUs should be adopted. This will make SUs quickly run out of battery. On the contrary, densely deployed CRrouters will not only incur higher capital expenditure (CAPEX) and operating expense (OPEX), but also increase coordination complexity. Moreover, the deployment of CR-routers is highly related to spectral efficiency. According to the distances among different CR-routers, spectrum availability shows different levels of correlation. Intuitively, when getting closer, two CR-routers will have higher possibility to share similar available bands, which improves the utilization of harvested bands. However, to coordinate those densely deployed CR-routers, we might need to allocate more basic bands for control signaling, which results in fewer basic bands for data services. Thus, how to economically deploy CR-routers to efficiently utilize available resources while meeting enough service demands and also conserving energy is an interesting and challenging problem. We notice that there are multiple recent works on the infrastructure node placement problem in CRNs. Li et al. [110] study the placement of secondary access points for two competing operators as well as the corresponding user association process by formulating a power-discrimination spatial game. Zhu et al. [111] find the optimal relay location for a two-hop CRN subject to outage constraints. Unfortunately, the features of the CCHN, mesh-typed wireless backhaul and SUs' heterogeneous communication devices, are not considered in these works. In the CCHN, how to deploy CR-routers will not only relate to how data traffic are routed but also how spectrum resources are allocated in the cognitive radio mesh. This deployment problem will be further complicated by the broadcast nature of the wireless medium and the limited battery storage of SUs' devices. As the activities of multiple CR-routers might be affected by the same PU, available spectrum bands in different links in the cognitive radio mesh are correlated. Since SUs' devices might be battery-powered, CR-routers should be deployed such that the energy consumption of SUs' devices are constrained below certain level. That is, when deploying CR-routers in the CCHN, we should jointly consider the distribution of SUs' traffic requests, PUs' activities and distributions and the energy consumption of SUs' devices, which makes the CR-router placement problem in the CCHN different and challenging. Although we have obtained some preliminary results on this problem with simplifying assumption in [112], substantial amount of work is still necessary.

\section{Cross Layer Support for SUs Under Uncertain Spectrum Supply}

Instead of single-hop transmissions as most commonly studied in current cognitive radio research, data traffic in our CCHN may be delivered in a multi-hop fashion. The capability of multi-hop transmissions in a cognitive radio mesh network makes our CCHN more flexible and paves the way for network capacity enhancement. Multi-hop transmissions bring about many advantages, such as reduced transmit power, more aggressive frequency reuse, better exploitation of locally available spectrum bands, and load balancing. To exploit these advantages, we need to cope with several challenges. As aforementioned, a cognitive radio mesh needs to support multiple sessions with distinct sources and destinations. Since the data flows of these sessions come with different rates and QoS constraints, the flow paths should be carefully designed so that more sessions could be supported. Clearly, it is impossible to achieve flow optimization without considering the throughput provided by different links which is related to link scheduling and resource allocation. Thus, when allocating spectrum resources for flow optimization in a cognitive radio mesh, we need to utilize cross-layer information to jointly consider routing, scheduling and resource allocation. As CR-routers have cognitive radio capability, when performing network optimization, we should also consider how to efficiently utilize unused licensed spectrum bands. For example, reduced transmit power might lead to the decrease of data rate on a specific channel, but will also make more PUs' spectrum bands available through frequency reuse. Thus, the CCHN could support more sessions via regulating the transmit power of 
CR-routers. It should be noted that harvested spectrum bands are not reliable due to the unexpected return of PUs. This uncertainty makes it impossible to offer a strict QoS guarantee on harvested spectrum bands. This is why we push delay-tolerant data to harvested bands and still support delay sensitive traffic in SSP's basic bands. Due to the uncertainty in PUs' spectrum bands, the network optimization, i.e., routing, link scheduling and resource allocation, in $\mathrm{CCHN}$, is highly interesting but challenging. In this case, the techniques used for traditional multi-channel and multi-radio systems are not applicable and stochastic optimization seems to be the only way out [67]-[69], [83], [91], [113]. Another possible method may be the quantile/superquantile optimization [114], which shares the same spirit as our optimization approach as alluded above. To ensure effective statistical QoS provisioning, we also need to find an effective stochastic model to capture the random nature of the uncertain harvested spectrum resources, which is not an easy task considering the spatial and temporal variations in PUs' activities and requires more research efforts.

\section{Fine Grained Spectrum Mapping}

To efficiently use the spectrum and regulate service provisioning, the SSP has to maintain an accurate and high resolution spectrum usage map within its coverage area. To get high-quality spectrum statistics, a fundamental problem is how often spectrum sensing should be performed via CR-routers. Too frequent spectrum sensing will introduce too much overhead and energy consumption. Particularly, when cooperative spectrum sensing is adopted, frequent sensing activities will introduce large amounts of communication overhead. On the other hand, with less frequent spectrum sensing, the SSP, BSs and CR-routers could not immediately be aware of variations in spectrum availability, which will lead to harmful interference to PUs as well as inefficient spectrum utilization. Another problem concerning spectrum sensing is how to coordinate CR-routers and spectrum sensing nodes to get a fine grained spectrum mapping. Since spectrum availability is location-dependent and time-varying, the number of spectrum sensing nodes, the locations of fixed sensing nodes, and the path planning of mobile sensing nodes are all needed to be carefully investigated to provide an accurate, comprehensive and timely-updated spectrum map. Since spectrum sensing algorithms and devices are the basis of the fine grained spectrum mapping, research efforts should still be devoted to developing more efficient spectrum sensing algorithms and devices (i.e., CR-routers), which are still under investigation.

\section{E. Traffic Analysis}

In the $\mathrm{CCHN}$, data services are handled differently based on whether they are delay-sensitive or delay-tolerant. To achieve this goal, we need to know what kinds of data are delay-sensitive and what kinds of data are delaytolerant. Unfortunately, this is not an easy task. The network performance will be significantly affected by the classification. If the criteria is too conservative, only limited amount of traffic will be considered as delay-tolerant, which results in limited performance gain. When the classification is too aggressive, too much traffic will be pushed to harvested bands, which will cause congestion and lead to performance degradation. More importantly, whether a data service is delay-sensitive or delay-tolerant not only depends on the property of the application, e.g., its data volumes and delay constraints, but also depends on the capability of the considered network. A data service, which is delay-tolerant in a network with high data rate, might become delay-sensitive in a network with limited bandwidth. To determine what kinds of traffic could be considered as delay tolerant, we should collect huge amount of network statistics and conduct an extensive and comprehensive analysis. Thus, teletraffic analysis should be revisited for newly emerging mobile applications. Although we have witnessed constantly growing interests in mobile traffic analysis, these works are mainly focused on extracting and modelling spatial-temporal traffic patterns within the telecommunication networks [115]-[117]. As pointed out in [117], efficient techniques to simultaneously capture the three dimensions of teletraffic, i.e., space, time and the type of services, are still lacking. In view of this, traditional traffic engineering for cellular networks may not be enough and a revisit to teletraffic analysis of newly emerging services consisting of various delay-tolerant services is necessary.

\section{F. Spectrum Allocation for Control and Data Plane: C/U Decoupling}

Efficient resource utilization in CRNs is achieved through centralized control of the SSP. In the CCHN, the SSP employs CR-routers to collect information on spectrum availability and traffic distribution in its service area to facilitate network optimization. Once routing, link scheduling and resource allocation have been determined, the SSP sends these decisions back to the CR-routers to coordinate their activities. Obviously, this process involves a lot of control signaling exchanges between the SSP and CR-routers. Furthermore, each CRrouter needs to exchange control messages with SUs and other CR-routers to enable traffic distribution collection and multihop transmissions. To make the CCHN work properly and effectively, the SSP should allocate spectrum bands for these control information exchanges (C-plane). To ensure the CCHN function correctly, C-plane traffic should be handled with high reliability and strict delay constraints. As harvested bands are not reliable, C-plane traffic could only be conveyed through the basic bands. Due to limited amount of basic bands, more basic bands for control signaling implies fewer basic bands for delay sensitive data (U-plane) and SU access, which might lead to a decrease in system capacity. Therefore, how to optimally allocate precious basic bands for C-plane and U-plane traffics is of vital importance to the success of the CCHN. We have addressed this issue in [81] for wireless railway systems in which reliable low frequency bands are used to control the use of unreliable high frequency bands. Similar idea may be utilized for this design, which is still under research. ${ }^{3}$

\footnotetext{
${ }^{3}$ It should be noted that the concept of $\mathrm{C} / \mathrm{U}$ decoupling in the $\mathrm{CCHN}$ is slightly different from that in SDN. The $\mathrm{C} / \mathrm{U}$ decoupling in SDN aims to extract control functions from network devices in order to make networks more flexible [118], [119], while the C/U decoupling in the CCHN primarily results from the idea of employing reliable basic bands to supervise the utilization of unreliable harvested bands.
} 


\section{G. Channel Mapping and Frame Structure Redesign}

As mentioned previously, $\mathrm{C} / \mathrm{U}$ plane decoupling is applied in the CCHN to provide high-quality data services as well as efficient mobility support for SUs. C/U plane decoupling requires that control signaling and data traffic be handled over different frequency bands and possible different network entities, which is unfortunately not supported in current wireless networks, such as LTE networks. In LTE, C-plane and Uplane traffic are mixed in the physical layer even though they are separated logically in upper network layers. For example, although Dedicated Control Channel (DCCH) and Downlink Traffic Channel (DTCH) are different logic channels, they are carried on the same physical channel, i.e., Physical Downlink Shared Channel (PDSCH) [120]. To enable C/U plane decoupling in the CCHN, the mapping between logic channels and physical channels as well as the physical layer frame structures in current CRNs should be redesigned, which will be addressed in our CCHN.

\section{H. In-Network Caching}

In the CCHN, SUs' traffic and PUs' activities vary independently, which makes it difficult to efficiently utilize spectrum access opportunities. In some situations, the CCHN has more than enough unused licensed bands to support SUs' data traffic, while in other cases, SUs' data traffic is much higher than what the available spectrum bands could handle. It would be nice that the CCHN could take advantage of currently abundant spectrum resources to relieve latter congestion. In view of the continuous increase in content dissemination based services, such as video streaming services and video clips embedded in many mobile applications, in which users care more about data content rather than locations where these data are stored [121], [122], we could deploy caching techniques at the edge of the CCHN and utilize abundant harvested bands to proactively push data contents to the edge of the CCHN. Extensive caching could be implemented at CR-routers, or connected to CR-routers via wired connections. Later on, SUs' requests could be served by these cached content, which reduces the traffic in the cognitive radio mesh. In such a way, the CCHN is able to exploit current abundant spectrum resources to serve future massive traffic demands, which allows spectrum resources to be used more efficiently.

When proactive in-network caching is introduced to the $\mathrm{CCHN}$, a fundamental problem is where to cache data items. Due to limited capacity, a cache could not store all available data items. To improve SUs' experience, we must carefully study where each data item is cached. This problem is more challenging in the $\mathrm{CCHN}$, since the number of data items that could be delivered is limited by the availability of harvested spectrum bands in the $\mathrm{CCHN}$ which is highly dynamic in both spatial and temporal domains. An efficient caching scheme should jointly consider where and how to push those data items. This line of research can benefit from the study of information-centric networks (ICNs) [123]. We notice that similar problems have been addressed in a few recent works. For example, in-network caching is introduced to cognitive ad hoc networks based on the concept of named data networking for end-to-end data delivery in [124], and a caching placement problem for multi-hop CRNs is studied in [125] to balance the dissemination and access costs with constraints on data access delay. Unfortunately, it is still unclear how to efficiently utilize harvested bands for proactive in-network caching. During the proactive caching process, the intended content might be cached at the destined CR-router due to SUs' regular data requests. In addition, how to schedule the data traffic incurred by proactive caching and the operation of CR-routers, particularly considering uncertain PUs' activities, is still not well addressed. Although our recent work in [126] has solved this problem to some extent, considerable research efforts are still needed.

\section{Spectrum Auction}

As SSPs are involved in spectrum auction, existing works on per-user based auction could not be directly applied and we need to revisit both auction mechanism design as well as SSPs' bidding strategies to make spectrum auction effective [127]-[129]. Generally speaking, an auction mechanism consists of winner determination mechanism, which determines the winners of an auction, and payment mechanism, which specifies how the winners are charged [127]-[129]. Noticing spectrum reusability, a group of buyers without mutual interference are usually claimed as winners, which implies winner determination in spectrum auction is closely related to interfering/conflicting relationships among buyers [127], [130], [131]. Since different SSPs might deploy their BSs and CR-routers in overlapped areas, the conflicting relations among SSPs are more complicated than those in per-user based spectrum auction. In this case, how to determine the winners is very challenging but interesting. Once winners are determined, we need a payment mechanism to guarantee the auction mechanism is truthful. As mentioned in Section III-F, each SSP might require multiple bands in different areas to support its services and thus must bid for a group of spectrum bands simultaneously. To make spectrum auction more effective, the auctioneer might allow each SSP to bid for multiple groups of spectrum bands as what has been done in combinatorial auction [128]. Unfortunately, this will introduce competition among those bids from the same SSP. Under this circumstance, how to design a payment mechanism to make spectrum auction truthful is very interesting. Given the auction mechanism, each SSP will determine their bidding strategies accordingly. Unlike per-user based auction, SSPs bid for extra spectrum bands to serve their associated SUs. Although each SSP could support more SUs with more spectrum resources, it is more likely to cause conflicts with other SSPs by bidding for more spectrum bands [67], [69], [127]. Thus, the determination of bidding strategies for SSPs is more involved than that in per-user based auction since they need to jointly consider resource allocation, SUs' bids and the cost of required spectrum bands.

\section{Selected Application Scenarios}

The flexibility of our CCHN renders itself numerous potential applications. In many practical situations, many 
functionalities of CR-routers and BSs could be fulfilled by or easily incorporated into existing network entities, which makes it easier to upgrade existing wireless networks with cognitive radio technology. Moreover, since CR-routers are wirelessly backhauled to BSs, the proposed CCHN not only eases network deployment, but also more effectively takes advantage of existing infrastructure. In what follows, we will discuss some potential application scenarios in which our $\mathrm{CCHN}$ provides perfect fit for network service provisioning.

\section{A. Cellular Networks}

Recent popularity of smart devices leads to a sudden surge of various mobile applications, such as anywhere anytime online social networking and mobile video services, which results in an explosive growth of mobile data traffic. Unfortunately, current allocated cellular bands are far from enough to support such a huge amount of data traffic and will soon get congested [1]. To address this problem, one traditional solution is to add more bandwidth, which would be a hard thing to do. An alternative solution is to search for others' bandwidth and harvest unused (licensed/unlicensed) bands to take care of some cellular loads, resulting in cognitive cellular networks (CCNs) [38], [107], [132], [133]. A fundamental problem here is how to implement the $\mathrm{CCN}$. The natural questions first to ask are what kinds of spectrum bands to acquire and what kinds of traffic should to support with these harvested bands. Since harvested bands have to be evacuated when PUs of a licensed band returns, it is hard to support QoS stringent services. Fortunately, many mobile applications, such as video clips, best effort data services, some short messages, upload/download file services, etc., are delay tolerant and could be effectively supported with opportunistic use of other bands. Thus, the cellular operator could offload its delay tolerant traffic to the harvested bands to save cellular bands for more delay-sensitive services. Moreover, to be cost-effective and enable rapid deployment, the envisioned $\mathrm{CCN}$ should be flexible so that existing cellular networks could be easily integrated with the CCN.

The proposed CCHN naturally meets these requirements, particularly when the CSP is willing to upgrade its network with CCHN capability. In this case, the CSP serves as the SSP with its own basic cellular bands. It could modify its BSs, including marco BSs (MBSs) and Small BSs (SBSs), to endow them with cognitive radio capability and wireless routing capability. Thus, the cellular BSs with wired backhaul work as BSs in the CCHN, while others, such as SBSs or access points (APs), work as CR-routers and collectively form a cognitive radio mesh [38], [133], [134]. The CSP might also deploy some additional fixed or mobile CR-routers in its service area. These mobile CR-routers could be fast deployed at hot spots to provide services or could physically deliver data between two CR-routers/BSs via the store-carry-forward technique [135]. Cellular users are served by nearby CRrouters/BSs depending on their proximities. Each CR-router aggregates data requests in its coverage area and submits the aggregated requests to the CSP. Due to this aggregation, the conflicts in control channels can be greatly reduced

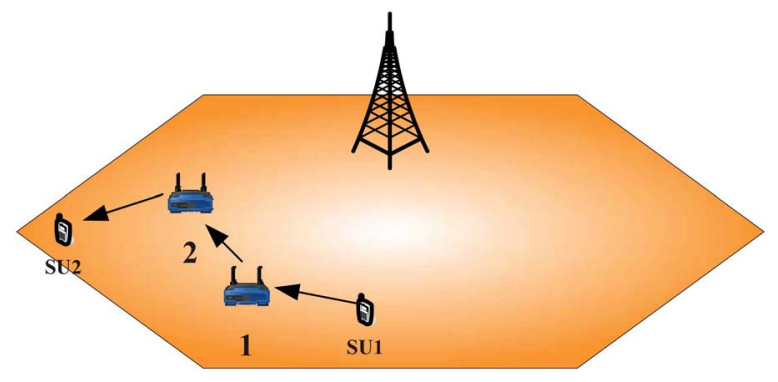

Fig. 6. An illustrative example for D2D communications in the CCHN.

and cellular networks could accommodate more connections. Furthermore, the CSP, i.e., the SSP, could configure these CRrouters to deliver data to BSs via multi-hop transmissions. For example, once a hot spot is detected, the CSP could reconfigure its CR-routers to direct delay tolerant data to lightly loaded areas via harvested bands, which not only relieves congestion, but also improves user experience. Additionally, with the help of cognitive radio mesh, the CSP could push delay tolerant data via harvested bands to the edge of the cellular network where cellular users enjoy high speed access with low energy consumption due to closer proximities, which can also reduce co-channel interference. Another benefit with this kind of data delivery mechanism is the frequency efficiency increase due to improved frequency reuse of cellular bands. Some channels within traditional frequency reuse distance may be borrowed due to lower transmission power between cellular users and edge CR-routers. With the cognitive radio mesh in place, the SSP is able to take advantage of multi-hop device-to-device (D2D) communication in the sense that cellular users' data are directly exchanged without going through the BS or data networks [136]-[138]. For example, as shown in Fig. 6, the SSP can facilitate data transmissions between SU1 and SU2 via $\mathrm{CR}$-router 1 and 2. For non-CR devices, their associated CR-routers/BSs can tune to the basic cellular bands to deliver services, while other cellular devices could employ both cellular bands and harvested bands for communications. In such a way, cellular users could take advantage of cognitive radio technology even if they do not have cognitive radio capability. Another benefit of the CCHN is the possible price reduction on the cellular data charge, as most of cellular data are delivered via harvested bands, which are relatively cheaper than the cellular bands. If spectrum auction can be made practical, the cellular operators could interact with spectrum market and purchase enough bandwidth to support their offloading services. They could only design viable service provisioning to address the end-to-end QoS by jointly managing their own licensed bands and harvested bands because they have full view of service requests and resource availability. Just because the cellular operators have already addressed their security and privacy issues over their own systems, they could easily addressed the security and privacy over the cognitive radio segments. In this sense, the proposed cognitive radio enhancement to existing cellular systems pave the way to address the potential congestion and spectrum shortage problems without adding licensed bandwidth. 


\section{B. Disaster Relief and Rescue}

Unexpected natural/man-made disasters, such as earthquake or terrorist attacks, may cause severe damage and possibly significant loss of life. During the disasters, communication infrastructures might be fully/partially destroyed, and there may cause lack of communication connections in the impacted areas. As reported in [139], after the Wenchuan earthquake in China, 28, 714 mobiles and PHS base stations were destroyed and the worst affected areas experienced an outage of communication connections. In contrast, there will be a surge in communication traffic since victims may attempt to search for help in all possible ways while first responders need to report/coordinate their activities. The limited remaining network resources may soon become congested, which hinders the relief and rescue efforts. To provide communication services in the affected areas, a flexible network, which could be rapidly deployed and efficiently utilizes all available network resources, is of vital importance. In this situation, the CCHN architecture could be utilized to build up an emergency communication network.

One possible viable solution is to utilize emergency communication vehicles and disaster relief vehicles quickly deployed in the affected areas after disasters. The emergency communication vehicles (ECVs) serve as BSs and could be connected to core networks through various kinds of methods, such as satellite communication [140]. The disaster relief vehicles are equipped with communication devices with cognitive radio capability. They serve as either mobile or static CR-routers and form a cognitive radio mesh where data traffic is forwarded through multi-hop transmissions. For inaccessible areas, we could airdrop CR-routers by helicopters [139]. As in the CCHN, the operations of the CR-routers are supervised by the CSP via either ECVs or BSs still in place. All these network components, including the vehicles and the BSs, could transmit in both cellular bands and harvested bands. Meanwhile, the first responders could be equipped with handheld devices which could collaborate with CR-routers to sense for available spectrum and build up the spectrum map. Additionally, emergency vehicles could be used as data mules [141] to carry delay tolerant data, such as video records and pictures of the hit area.

The above proposed emergency communication network shares all advantages of the CCHN. Instead of directly communicating with ECVs or BSs, mobile users, such as victims and first responders, could connect to the nearby CR-routers due to the fact that CR-routers could tune to the radio interface of affected users and establish immediate communications. CRrouters aggregate users' traffic and forward these data through multi-hop transmissions. Aggregation at CR-routers relieves the conflicts in control channels and multi-hop transmissions improves spectrum reuse. Moreover, this architecture benefits mobile devices with limited batteries in post-disaster situations. After a disaster, it might be difficult for mobile devices to get recharged since infrastructures and facilities may be ruined. However, as mobile devices may have to connect to remote BSs to get services, which makes batteries drain quickly. By connecting to CR-routers, the energy in mobile devices could be saved for more important use. Furthermore, CR-routers could actively search for available spectrum bands and push delay tolerant data to harvested bands, which not only boost system capacity, but also benefit users without cognitive radio capability. The CR-routers could also be used for intelligence harvesting, such as the pictures of hit areas, victim distributions, and weather conditions. All the collected information can be processed and sent to disaster control center for coordination and control. Meanwhile, the CR-routers could be used to broadcast information such as battery charging stations, the locations of refuges, and potential secondary disasters.

\section{Mobile Health}

Mobile health (mHealth) is seen as a cost-effective way to deliver healthcare services, particularly to places lacking healthcare facilities [142]-[144]. By utilizing mobile devices and wireless communication technologies, mHealth enables long-term and continuous health monitoring for patients particularly for those who have chronic diseases. In mHealth systems, various kinds of biomedical sensors are deployed on people's bodies to collect vital bio-signals and physiological parameters, such as electrocardiogram, blood pressure, heart rate and body temperature [142]. The collected data are forwarded to possibly mobile data collection or processing devices through wireless connections. Mobile devices either locally process these data or send them to remote servers for further processing and analysis. When an abnormal or emergency situations are detected, requests are sent to alert caregivers/doctors for assistance [142].

The success of mHealth heavily relies on the capability of mobile devices and communications technologies. The delivery of mHealth services involves frequent and huge amount of data transmissions between mobile devices and remote servers, which may not be effectively handled if merely based on traditional telecommunications systems such as cellular networks. On the one hand, mobile devices are often with limited battery power and may quickly run out of energy due to frequent communication with BSs [144]. On the other hand, with frequent and huge amount of data transmission, traditional telecommunications networks may soon get congested due to limited capacity. In particular, the traffic on control channels will significantly increase as mobile devices need to regularly report vital biomedical signals and parameters for continuous monitoring and timely detection [142], [145]. Fortunately, our proposed CCHN is a promising way to deal with these challenges and enables us to fully enjoy the benefits of mHealth. As presented in Section $\mathrm{V}-\mathrm{A}$, once the CSP upgrades its network to integrate CCHN capability, mobile devices could directly connect to nearby CR-routers instead of BSs, which not only saves energy, but also mitigates the congestions in both control and data channels. More importantly, CR-routers are able to harvest and utilize unused licensed bands for transmissions, and data could be carried on either licensed bands or harvested bands according to the traffic types. Specifically, delay tolerant data, such as non-critical healthcare data for disease prevention, can be pushed to unreliable harvested bands to save the precious reliable licensed bands for delay sensitive traffic, such as 
assistance requests in case of emergency and data generated from real time monitoring for life critical situations. In such a way, huge amount of data can be transmitted on cheaper harvested bands rather than on expensive licensed bands, which could reduce the cost of mHealth applications and contribute to the wide deployment of mHealth systems. The cloud-assisted remote monitoring technology we developed in [146] may be incorporated into our CCHN for healthcare applications in which huge data transportations are needed.

\section{CONCLUSION}

Various kinds of mobile data arising from the wide applications of smart devices, such as smart phones and sensing devices, demands a flexible and effective cognitive radio network (CRN) architecture to efficiently utilize communications and network resources. In this paper, we have presented a tutorial on how such a novel flexible network architecture for future cognitive radio technologies can be designed with the cognitive capacity harvesting network $(\mathrm{CCHN})$ as an example. We have provided a comprehensive review on existing architectural design issues in the current CRNs and identified problems hindering the adoption of CRNs in practice. Based on our analysis, we have concluded that a successful CRN should be able to benefit non-CR capable devices, establish reliable common control channels, efficiently utilize network resources, provide network-side support for spectrum auction, and guarantee statistical end-to-end (e2e) QoS. To achieve these goals, we have introduced an SSP, which is an independent wireless service provider with its own basic (licensed) bands, and CR-routers, which forms a cognitive radio mesh as backhaul facilities to enable effective information collection and efficient resource allocation. We have demonstrated that our proposed $\mathrm{CCHN}$ can address various design challenges in existing CRNs and thus provides a very promising solution to future CRNs. Although our CCHN has many nice features, certain research efforts are still needed to fully exploit the advantages of the CCHN. We hope this paper could stimulate more research efforts to not only improve the CCHN, but also yield viable approaches to implementing CRNs.

\section{REFERENCES}

[1] "Cisco visual networking index: Global mobile data traffic forecast update, 2015-2020," White Paper, Cisco, San Jose, CA, USA, Feb. 2016.

[2] NSF Leads Federal Effort to Boost Advanced Wireless Research, NSF, Arlington, VA, USA, Jul. 2016. [Online]. Available: http://www.nsf.gov/news/news_summ.jsp?cntn_id=139179\&org= NSF\& from $=$ news

[3] T. M. Cover and J. A. Thomas, Elements of Information Theory, 2nd ed. New York, NY, USA: Wiley, 2006.

[4] C. D. Bazelon and G. McHenry, "Substantial licensed spectrum deficit (2015-2019): Updating the FCC's mobile data demand projections," prepared for CTIA-The Wireless Association, Washington, DC, USA, Jun. 2015.

[5] S. M. Mishra et al., "A real time cognitive radio testbed for physical and link layer experiments," in Proc. IEEE DySPAN, Baltimore, MD, USA, Nov. 2005, pp. 562-567.

[6] T. M. Taher, R. B. Bacchus, K. J. Zdunek, and D. A. Roberson, "Longterm spectral occupancy findings in Chicago," in Proc. IEEE DySPAN, Aachen, Germany, May 2011, pp. 100-107.

[7] FCC Adopts Rules to Facilitate Next Generation Wireless Technologies, FCC, Washington, DC, USA, Jul. 2016. [Online]. Available: https://www.fcc.gov/document/fcc-adopts-rules-facilitate-nextgeneration-wireless-technologies
[8] R. Krause. "AT\&T, Verizon Get Their Wish: FCC Opens Up $5 G$ Airwaves. Accessed on Jul. 14, 2016. [Online]. Available: http://www.investors.com/news/technology/att-verizon-gettheir-wish-fcc-opens-up-5g-airwaves/

[9] S. Haykin, "Cognitive radio: Brain-empowered wireless communications," IEEE J. Sel. Areas Commun., vol. 23, no. 2, pp. 201-220, Feb. 2005.

[10] I. F. Akyildiz, W.-Y. Lee, M. C. Vuran, and S. Mohanty, "Next generation/dynamic spectrum access/cognitive radio wireless networks: A survey," Comput. Netw., vol. 50, no. 13, pp. 2127-2159, Sep. 2006.

[11] A. Ali and W. Hamouda, "Advances on spectrum sensing for cognitive radio networks: Theory and applications," IEEE Commun. Surveys Tuts., to be published.

[12] S. K. Sharma, E. Lagunas, S. Chatzinotas, and B. Ottersten, "Application of compressive sensing in cognitive radio communications: A survey," IEEE Commun. Surveys Tuts., vol. 18, no. 3, pp. 1838-1860, 3rd Quart., 2016.

[13] S. K. Sharma et al., "Cognitive radio techniques under practical imperfections: A survey," IEEE Commun. Surveys Tuts., vol. 17, no. 4, pp. 1858-1884, 4th Quart., 2015.

[14] Y. Xu, X. Zhao, and Y.-C. Liang, "Robust power control and beamforming in cognitive radio networks: A survey," IEEE Commun. Surveys Tuts., vol. 17, no. 4, pp. 1834-1857, 4th Quart., 2015.

[15] M. T. Masonta, M. Mzyece, and N. Ntlatlapa, "Spectrum decision in cognitive radio networks: A survey," IEEE Commun. Surveys Tuts., vol. 15, no. 3, pp. 1088-1107, 3rd Quart., 2013.

[16] L. Gavrilovska, V. Atanasovski, I. Macaluso, and L. A. DaSilva, "Learning and reasoning in cognitive radio networks," IEEE Commun. Surveys Tuts., vol. 15, no. 4, pp. 1761-1777, 4th Quart., 2013.

[17] M. Bkassiny, Y. Li, and S. K. Jayaweera, "A survey on machinelearning techniques in cognitive radios," IEEE Commun. Surveys Tuts., vol. 15, no. 3, pp. 1136-1159, 3rd Quart., 2013.

[18] Y. Chen and H.-S. Oh, "A survey of measurement-based spectrum occupancy modeling for cognitive radios," IEEE Commun. Surveys Tuts., vol. 18, no. 1, pp. 848-859, 1st Quart., 2016.

[19] E. Z. Tragos, S. Zeadally, A. G. Fragkiadakis, and V. A. Siris, "Spectrum assignment in cognitive radio networks: A comprehensive survey," IEEE Commun. Surveys Tuts., vol. 15, no. 3, pp. 1108-1135, 3rd Quart., 2013.

[20] G. I. Tsiropoulos, O. A. Dobre, M. H. Ahmed, and K. E. Baddour, "Radio resource allocation techniques for efficient spectrum access in cognitive radio networks," IEEE Commun. Surveys Tuts., vol. 18, no. 1, pp. 824-847, 1st Quart., 2016.

[21] E. Ahmed, A. Gani, S. Abolfazli, L. J. Yao, and S. U. Khan, "Channel assignment algorithms in cognitive radio networks: Taxonomy, open issues, and challenges," IEEE Commun. Surveys Tuts., vol. 18, no. 1, pp. 795-823, 1st Quart., 2016.

[22] L. Gavrilovska, D. Denkovski, V. Rakovic, and M. Angjelichinoski, "Medium access control protocols in cognitive radio networks: Overview and general classification," IEEE Commun. Surveys Tuts., vol. 16, no. 4, pp. 2092-2124, 4th Quart., 2014.

[23] Y. Zhang, C. Lee, D. Niyato, and P. Wang, "Auction approaches for resource allocation in wireless systems: A survey," IEEE Commun. Surveys Tuts., vol. 15, no. 3, pp. 1020-1041, 3rd Quart., 2013.

[24] M. E. Tanab and W. Hamouda, "Resource allocation for underlay cognitive radio networks: A survey," IEEE Commun. Surveys Tuts., to be published.

[25] M. Naeem, A. Anpalagan, M. Jaseemuddin, and D. C. Lee, "Resource allocation techniques in cooperative cognitive radio networks," IEEE Commun. Surveys Tuts., vol. 16, no. 2, pp. 729-744, 2nd Quart., 2014.

[26] M. Youssef, M. Ibrahim, M. Abdelatif, L. Chen, and A. V. Vasilakos, "Routing metrics of cognitive radio networks: A survey," IEEE Commun. Surveys Tuts., vol. 16, no. 1, pp. 92-109, 1st Quart., 2014.

[27] R. K. Sharma and D. B. Rawat, "Advances on security threats and countermeasures for cognitive radio networks: A survey," IEEE Commun. Surveys Tuts., vol. 17, no. 2, pp. 1023-1043, 2nd Quart., 2015.

[28] L. Zhang et al., "Byzantine attack and defense in cognitive radio networks: A survey," IEEE Commun. Surveys Tuts., vol. 17, no. 3, pp. 1342-1363, 3rd Quart., 2015.

[29] X. Huang, T. Han, and N. Ansari, "On green-energy-powered cognitive radio networks," IEEE Commun. Surveys Tuts., vol. 17, no. 2, pp. 827-842, 2nd Quart., 2015.

[30] Y.-C. Liang, K.-C. Chen, G. Y. Li, and P. Mahonen, "Cognitive radio networking and communications: An overview," IEEE Trans. Veh. Technol., vol. 60, no. 7, pp. 3386-3407, Sep. 2011.

[31] S. Sengupta and K. P. Subbalakshmi, "Open research issues in multi-hop cognitive radio networks," IEEE Commun. Mag., vol. 51, no. 4, pp. 168-176, Apr. 2013. 
[32] A. M. Wyglinski, M. Nekovee, and T. Hou, Cognitive Radio Communications and Networks: Principles and Practice. San Diego, CA, USA: Academic Press, 2010.

[33] S. Srikanteswara and D. Choudhury, "A review of TV whitespace portable devices," in Proc. IEEE RWS, New Orleans, LA, USA Jan. 2010, pp. 480-483.

[34] S. J. Shellhammer, A. K. Sadek, and W. Zhang, "Technical challenges for cognitive radio in the TV white space spectrum," in Proc. Inf. Theory Appl. Workshop, San Diego, CA, USA, Feb. 2009, pp. 323-333.

[35] G. Hattab and M. Ibnkahla, "Multiband spectrum access: Great promises for future cognitive radio networks," Proc. IEEE, vol. 102, no. 3, pp. 282-306, Mar. 2014.

[36] H. Li and Z. Han, "Dogfight in spectrum: Combating primary user emulation attacks in cognitive radio systems, part I: Known channel statistics," IEEE Trans. Wireless Commun., vol. 9, no. 11, pp. 3566-3577, Nov. 2010.

[37] M. Pan, J. Sun, and Y. Fang, "Purging the back-room dealing: Secure spectrum auction leveraging Paillier cryptosystem," IEEE J. Sel. Areas Commun., vol. 29, no. 4, pp. 866-876, Apr. 2011.

[38] M. Li, P. Li, X. Huang, Y. Fang, and S. Glisic, "Energy consumption optimization for multihop cognitive cellular networks," IEEE Trans. Mobile Comput., vol. 14, no. 2, pp. 358-372, Feb. 2015.

[39] Y. Song, C. Zhang, and Y. Fang, "Stochastic traffic engineering in multihop cognitive wireless mesh networks," IEEE Trans. Mobile Comput., vol. 9, no. 3, pp. 305-316, Mar. 2010.

[40] B. Farhang-Boroujeny, "Filter bank spectrum sensing for cognitive radios," IEEE Trans. Signal Process., vol. 56, no. 5, pp. 1801-1811, Apr. 2008.

[41] L. Duan, J. Huang, and B. Shou, "Investment and pricing with spectrum uncertainty: A cognitive operator's perspective," IEEE Trans. Mobile Comput., vol. 10, no. 11, pp. 1590-1604, Nov. 2011.

[42] S. Wang, F. Tosato, and J. P. Coon, "Reliable energy-efficient spectrum management and optimization in cognitive radio networks: How often should we switch?" IEEE Wireless Commun., vol. 20, no. 6, pp. 14-20, Dec. 2013.

[43] S. Wang, K. Mimis, M. Z. Bocus, G. T. Watkins, and J. P. Coon, "Cognitive antenna selection relay for green heterogeneous healthcare networks," IEEE Wireless Commun., vol. 20, no. 5, pp. 44-52, Nov. 2013.

[44] Y. Tawk, J. Costantine, and C. G. Christodoulou, "Cognitive-radio and antenna functionalities: A tutorial [wireless corner]," IEEE Antennas Propag. Mag., vol. 56, no. 1, pp. 231-243, Feb. 2014.

[45] J. H. Saltzer, D. P. Reed, and D. D. Clark, "End-to-end arguments in system design," ACM Trans. Comput. Syst., vol. 2, no. 4, pp. 277-288, 1984.

[46] A. Leon-Garcia and I. Widjaja, Communication Networks Fundamental Concepts and Key Architectures. London, U.K.: McGraw-Hill, 2004.

[47] G. A. Shah and O. B. Akan, "Cognitive adaptive medium access control in cognitive radio sensor networks," IEEE Trans. Veh. Technol., vol. 64, no. 2, pp. 757-767, Feb. 2015.

[48] K. G. M. Thilina, E. Hossain, and D. I. Kim, "DCCC-MAC: A dynamic common control channel-based MAC protocol for cellular cognitive radio networks," IEEE Trans. Veh. Technol., vol. 65, no. 5, pp. 3597-3613, May 2016.

[49] L. Li and C. Xu, "On ergodic sum capacity of fading channels in OFDMA-based cognitive radio networks," IEEE Trans. Veh. Technol., vol. 63, no. 9, pp. 4334-4343, Nov. 2014.

[50] Y. Long, H. Li, H. Yue, M. Pan, and Y. Fang, "SUM: Spectrum utilization maximization in energy-constrained cooperative cognitive radio networks," IEEE J. Sel. Areas Commun., vol. 32, no. 11, pp. 2105-2116, Nov. 2014.

[51] Z. Gu, Q.-S. Hua, and W. Dai, "Fully distributed algorithms for blind rendezvous in cognitive radio networks," in Proc. MobiHoc, Philadelphia, PA, USA, Aug. 2014, pp. 155-164.

[52] L. Yu, H. Liu, Y.-W. Leung, X. Chu, and Z. Lin, "Multiple radios for fast rendezvous in cognitive radio networks," IEEE Trans. Mobile Comput., vol. 14, no. 9, pp. 1917-1931, Sep. 2015.

[53] X. Hong, J. Wang, C.-X. Wang, and J. Shi, "Cognitive radio in 5G: A perspective on energy-spectral efficiency trade-off," IEEE Commun. Mag., vol. 52, no. 7, pp. 46-53, Jul. 2014.

[54] P. D. Mankar, G. Das, S. S. Pathak, and R. V. Rajakumar, "A method for accessing spatial spectrum holes for relay based cognitive cellular networks," IEEE Wireless Commun. Lett., vol. 4, no. 3, pp. 245-248, Jun. 2015.

[55] S. A. Ahmad and L. A. DaSilva, "Power control and soft topology adaptations in multihop cellular networks with multi-point connectivity," IEEE Trans. Commun., vol. 63, no. 3, pp. 683-694, Mar. 2015.
[56] X. Yuan et al., "Toward transparent coexistence for multihop secondary cognitive radio networks," IEEE J. Sel. Areas Commun., vol. 33, no. 5 , pp. 958-971, May 2015.

[57] P. Li, X. Huang, and Y. Fang, "Capacity scaling of multihop cellular networks," in Proc. IEEE INFOCOM, Shanghai, China, Apr. 2011, pp. 2831-2839.

[58] J. Garcia-Rois et al., "On the analysis of scheduling in dynamic duplex multihop mmWave cellular systems," IEEE Trans. Wireless Commun., vol. 14, no. 11, pp. 6028-6042, Nov. 2015.

[59] Z. Han, R. Zheng, and H. V. Poor, "Repeated auctions with Bayesian nonparametric learning for spectrum access in cognitive radio networks," IEEE Trans. Wireless Commun., vol. 10, no. 3, pp. 890-900, Mar. 2011

[60] H. Li, C. Wu, and Z. Li, "Socially-optimal online spectrum auctions for secondary wireless communication," in Proc. IEEE INFOCOM, Hong Kong, Apr./May 2015, pp. 2047-2055.

[61] M. Khaledi and A. A. Abouzeid, "Dynamic spectrum sharing auction with time-evolving channel qualities," IEEE Trans. Wireless Commun., vol. 14, no. 11, pp. 5900-5912, Nov. 2015.

[62] M. Zandi, M. Dong, and A. Grami, "Dynamic spectrum access via channel-aware heterogeneous multi-channel auction with distributed learning," IEEE Trans. Wireless Commun., vol. 14, no. 11, pp. 5913-5926, Nov. 2015.

[63] Y. Zhu, B. Li, and Z. Li, "Designing two-dimensional spectrum auctions for mobile secondary users," IEEE J. Sel. Areas Commun., vol. 31, no. 3, pp. 604-613, Mar. 2013.

[64] L. H. Ungar, D. C. Parkes, and D. P. Foster, "Cost and trust issues in on-line auctions," in Proc. Workshop Agent Mediated Electron. Trading AMET, Minneapolis, MN, USA, May 1998, pp. 161-172.

[65] R. Cassady, Auctions and Auctioneering. Los Angeles, CA, USA: Univ. California Press, 1967.

[66] D. S. Palguna, D. J. Love, and I. Pollak, "Secondary spectrum auctions for markets with communication constraints," IEEE Trans. Wireless Commun., vol. 15, no. 1, pp. 116-130, Jan. 2016.

[67] M. Pan et al., "When spectrum meets clouds: Optimal session based spectrum trading under spectrum uncertainty," IEEE J. Sel. Areas Commun., vol. 32, no. 3, pp. 615-627, Mar. 2014.

[68] M. Pan, H. Yue, C. Zhang, and Y. Fang, "Path selection under budget constraints in multihop cognitive radio networks," IEEE Trans. Mobile Comput., vol. 12, no. 6, pp. 1133-1145, Jun. 2013.

[69] M. Pan, C. Zhang, P. Li, and Y. Fang, "Spectrum harvesting and sharing in multi-hop CRNs under uncertain spectrum supply," IEEE J. Sel. Areas Commun., vol. 30, no. 2, pp. 369-378, Feb. 2012.

[70] Q. Zhang and Y.-Q. Zhang, "Cross-layer design for QoS support in multihop wireless networks," Proc. IEEE, vol. 96, no. 1, pp. 64-76, Jan. 2008.

[71] L. Hanzo and R. Tafazolli, "A survey of QoS routing solutions for mobile ad hoc networks," IEEE Commun. Surveys Tuts., vol. 9, no. 2, pp. 50-70, 2nd Quart., 2007.

[72] H. Zhai, X. Chen, and Y. Fang, "Improving transport layer performance in multihop ad hoc networks by exploiting MAC layer information," IEEE Trans. Wireless Commun., vol. 6, no. 5, pp. 1692-1701, May 2007

[73] H. Zhai and Y. Fang, "Impact of routing metrics on path capacity in multirate and multihop wireless ad hoc networks," in Proc. IEEE Int. Conf. Netw. Protocols (ICNP), Santa Barbara, CA, USA, Nov. 2006, pp. 86-95.

[74] H. Zhai, J. Wang, and Y. Fang, "Providing statistical QoS guarantee for voice over IP in the IEEE 802.11 wireless LANs," IEEE Wireless Commun., vol. 13, no. 1, pp. 36-43, Feb. 2006.

[75] H. Zhai and Y. Fang, "Distributed flow control and medium access in multihop ad hoc networks," IEEE Trans. Mobile Comput., vol. 5, no. 11, pp. 1503-1514, Nov. 2006.

[76] Y.-S. Su, S.-L. Su, and J.-S. Li, "Joint topology-transparent scheduling and QoS routing in ad hoc networks," IEEE Trans. Veh. Technol., vol. 63, no. 1, pp. 372-389, Jan. 2014.

[77] L. Cheng, J. Niu, J. Cao, S. K. Das, and Y. Gu, "QoS aware geographic opportunistic routing in wireless sensor networks," IEEE Trans. Parallel Distrib. Syst., vol. 25, no. 7, pp. 1864-1875, Jul. 2014.

[78] L. Hanzo, II, and R. Tafazolli, "QoS-aware routing and admission control in shadow-fading environments for multirate MANETs," IEEE Trans. Mobile Comput., vol. 10, no. 5, pp. 622-637, May 2011.

[79] X. Huang and Y. Fang, "Multiconstrained QoS multipath routing in wireless sensor networks," Wireless Netw., vol. 14, no. 4, pp. 465-478, Aug. 2008. 
[80] W. Chen, M. Sim, J. Sun, and C.-P. Teo, "From CVaR to uncertainty set: Implications in joint chance-constrained optimization," Oper. Res., vol. 58, no. 2, pp. 470-485, 2010.

[81] L. Yan, X. Fang, and Y. Fang, "Control and data signaling decoupled architecture for railway wireless networks," IEEE Wireless Commun., vol. 22, no. 1, pp. 103-111, Feb. 2015.

[82] Y. Fang and P. Li. NeTS: Collaborative Research: Cognitive Capacity Harvesting Networks, a Funded Project by National Science Foundation, 2011-2013. Accessed on Aug. 30, 2011. [Online]. Available: http://www.nsf.gov/ awardsearch/showAward?AWD_ID=1147813

[83] M. Pan, P. Li, Y. Song, Y. Fang, and P. Lin, "Spectrum clouds: A session based spectrum trading system for multi-hop cognitive radio networks," in Proc. IEEE INFOCOM, Orlando, FL, USA, Mar. 2012, pp. $1557-1565$.

[84] S. Sesia, I. Toufik, and M. P. J. Baker, LTE: The UMTS Long Term Evolution. Chichester, U.K.: Wiley, 2009.

[85] W. Tang, S. Feng, Y. Liu, and M. C. Reed, "Joint low-power transmit and cell association in heterogeneous networks," in Proc. IEEE Glob. Commun. Conf. (GLOBECOM), San Diego, CA, USA, 2015, pp. 1-6.

[86] H. S. Dhillon, R. K. Ganti, F. Baccelli, and J. G. Andrews, "Modeling and analysis of k-tier downlink heterogeneous cellular networks," IEEE J. Sel. Areas Commun., vol. 30, no. 3, pp. 550-560, Apr. 2012.

[87] D. Han et al., "Measurement and stochastic modeling of handover delay and interruption time of smartphone real-time applications on LTE networks," IEEE Commun. Mag., vol. 53, no. 3, pp. 173-181, Mar. 2015.

[88] H. Zhai, J. Wang, and Y. Fang, "DUCHA: A new dual-channel MAC protocol for multihop ad hoc networks," IEEE Trans. Wireless Commun., vol. 5, no. 11, pp. 3224-3233, Nov. 2006.

[89] Y. Moon et al., "Practicalizing delay-tolerant mobile apps with cedos," in Proc. 13th Annu. Int. Conf. Mobile Syst. Appl. Services, Florence, Italy, 2015, pp. 419-433.

[90] X. Li, H. Ding, M. Pan, Y. Sun, and Y. Fang, "Users first: Serviceoriented spectrum auction with a two-tier framework support," IEEE J. Sel. Areas Commun., vol. 34, no. 11, pp. 2999-3013, Nov. 2016.

[91] M. Pan, H. Yue, Y. Fang, and H. Li, "The X loss: Band-mix selection for opportunistic spectrum accessing with uncertain spectrum supply from primary service providers," IEEE Trans. Mobile Comput., vol. 11, no. 12, pp. 2133-2144, Dec. 2012.

[92] Spectrum Frontiers Order to Identify, Open Up Vast Amounts of New High-Band Spectrum for Next Generation (5G) Wireless Broadband, Federal Commun. Commission, Washington, DC, USA, Jun. 2016. [Online]. Available: https://www.fcc.gov/ document/rules-facilitate-next-generation-wireless-technologies

[93] J. Liu et al., "An energy-efficient strategy for secondary users in cooperative cognitive radio networks for green communications," IEEE $J$. Sel. Areas Commun., vol. 34, no. 12, pp. 3195-3207, Dec. 2016.

[94] D. M. Kalathil and R. Jain, "Spectrum sharing through contracts for cognitive radios," IEEE Trans. Mobile Comput., vol. 12, no. 10, pp. 1999-2011, Oct. 2013.

[95] L. Duan, L. Gao, and J. Huang, "Cooperative spectrum sharing: A contract-based approach," IEEE Trans. Mobile Comput., vol. 13, no. 1, pp. 174-187, Jan. 2014.

[96] L. Gao, L. Duan, and J. Huang, "Two-sided matching based cooperative spectrum sharing," IEEE Trans. Mobile Comput., vol. 16, no. 2, pp. 538-551, Feb. 2017

[97] E. Hossain, D. Niyato, and Z. Han, Dynamic Spectrum Access and Management in Cognitive Radio Networks. Cambridge, U.K.: Cambridge Univ. Press, 2009.

[98] "Reconfigurable radio systems (RRS); feasibility study on radio frequency (RF) performance for cognitive radio systems operating in UHF TV band white spaces," ETSI, Sophia Antipolis, France, Tech. Rep. ETSI TR 103 067, 2013.

[99] "Reconfigurable radio systems (RRS); cognitive radio system concept," ETSI, Sophia Antipolis, France, Tech. Rep. ETSI TR 102 802, 2010.

[100] A. Khattab and M. A. Bayoumi, "Standardization of cognitive radio networking: A comprehensive survey," Ann. Telecommun., vol. 70, nos. 11-12, pp. 465-477, Jun. 2015.

[101] M. Mueck et al., "ETSI reconfigurable radio systems: Status and future directions on software defined radio and cognitive radio standards," IEEE Commun. Mag., vol. 48, no. 9, pp. 78-86, Sep. 2010.

[102] S. Filin, H. Harada, H. Murakami, and K. Ishizu, "International standardization of cognitive radio systems," IEEE Commun. Mag., vol. 49, no. 3, pp. 82-89, Mar. 2011.
[103] M. Murroni et al., "IEEE 1900.6: Spectrum sensing interfaces and data structures for dynamic spectrum access and other advanced radio communication systems standard: Technical aspects and future outlook," IEEE Commun. Mag., vol. 49, no. 12, pp. 118-127, Dec. 2011.

[104] ECMA, "MAC and PHY for operation in TV white space," Standard ECMA-392, 2012

[105] "Introduction to cognitive radio systems in the land mobile service," Int. Telecommun. Union, Geneva, Switzerland, Tech. Rep. ITU-R M.2225, 2011.

[106] "Cognitive radio systems specific for international mobile telecommunications systems," Int. Telecommun. Union, Geneva, Switzerland, Tech. Rep. ITU-R M.2242, 2011.

[107] N. Zhang et al., "Cooperative heterogeneous framework for spectrum harvesting in cognitive cellular network," IEEE Commun. Mag., vol. 53 , no. 5, pp. 60-67, May 2015.

[108] K.-C. Chen, Y.-J. Peng, N. Prasad, Y.-C. Liang, and S. Sun, "Cognitive radio network architecture: Part I-General structure," in Proc. ACM ICUIMC, Suwon, South Korea, Jan. 2008, pp. 114-119.

[109] K.-C. Chen, P.-Y. Chen, N. Prasad, Y.-C. Liang, and S. Sun, "Trusted cognitive radio networking," Wireless Commun. Mobile Comput., vol. 10, no. 4, pp. 467-485, 2010.

[110] D. Li, W. Zhong, Q. Shi, H. Zhao, and B. Huang, "Secondary access points placement in cognitive radio networks: A spatial game mode with power discrimination," IEEE Trans. Veh. Technol., vol. 61, no. 6 , pp. 2729-2739, Jul. 2012 .

[111] J. Zhu, J. Huang, and W. Zhang, "Optimal one-dimensional relay placement in cognitive radio networks," in Proc. Int. Conf. Wireless Commun. Signal Process. (WCSP), Nanjing, China, 2010, pp. 1-6.

[112] H. Yue, M. Pan, Y. Fang, and S. Glisic, "Spectrum and energy efficient relay station placement in cognitive radio networks," IEEE J. Sel. Areas Commun., vol. 31, no. 5, pp. 883-893, May 2013

[113] Y. Long, H. Li, M. Pan, Y. Fang, and T. F. Wong, "A fair QoS-aware resource-allocation scheme for multiradio multichannel networks," IEEE Trans. Veh. Technol., vol. 62, no. 7, pp. 3349-3358, Sep. 2013.

[114] R. T. Rockafellar and S. Uryasev, "The fundamental risk quadrangle in risk management, optimization and statistical estimation," Surveys Oper. Res. Manag. Sci., vol. 18, nos. 1-2, pp. 33-53, 2013.

[115] F. Xu, Y. Li, H. Wang, P. Zhang, and D. Jin, "Understanding mobile traffic patterns of large scale cellular towers in urban environment," IEEE/ACM Trans. Netw., to be published.

[116] F. Xu et al., "Big data driven mobile traffic understanding and forecasting: A time series approach," IEEE Trans. Services Comput., vol. 9, no. 5, pp. 796-805, Sep./Oct. 2016

[117] D. Naboulsi, M. Fiore, S. Ribot, and R. Stanica, "Large-scale mobile traffic analysis: A survey," IEEE Commun. Surveys Tuts., vol. 18, no. 1, pp. 124-161, 1st Quart., 2016.

[118] S. Sezer et al., "Are we ready for SDN? Implementation challenges for software-defined networks," IEEE Commun. Mag., vol. 51, no. 7, pp. 36-43, Jul. 2013.

[119] F. A. Lopes, M. Santos, R. Fidalgo, and S. Fernandes, "A software engineering perspective on SDN programmability," IEEE Commun. Surveys Tuts., vol. 18, no. 2, pp. 1255-1272, 2nd Quart., 2016.

[120] E. Dahlman, S. Parkvall, and J. Sköld, 4G: LTE/LTE-Advanced for Mobile Broadband. Oxford, U.K.: Academic Press, 2011.

[121] B. Ahlgren, C. Dannewitz, C. Imbrenda, D. Kutscher, and B. Ohlman, "A survey of information-centric networking," IEEE Commun. Mag., vol. 50, no. 7, pp. 26-36, Jul. 2012.

[122] M. Zhang, H. Luo, and H. Zhang, "A survey of caching mechanisms in information-centric networking," IEEE Commun. Surveys Tuts., vol. 17, no. 3, pp. 1473-1499, 3rd Quart., 2015.

[123] H. Yue, L. Guo, R. Li, H. Asaeda, and Y. Fang, "DataClouds: Enabling community-based data-centric services over the Internet of Things," IEEE Internet Things J., vol. 1, no. 5, pp. 472-482, Oct. 2014.

[124] R. A. Rehman, J. Kim, and B.-S. Kim, "NDN-CRAHNs: Named data networking for cognitive radio ad hoc networks," Mobile Inf. Syst., vol. 2015, May 2015, Art. no. 281893.

[125] J. Zhao, W. Gao, Y. Wang, and G. Cao, "Delay-constrained caching in cognitive radio networks," IEEE Trans. Mobile Comput., vol. 15, no. 3, pp. 627-640, Mar. 2016.

[126] P. Si, H. Yue, Y. Zhang, and Y. Fang, "Spectrum management for proactive video caching in information-centric cognitive radio networks," IEEE J. Sel. Areas Commun., vol. 34, no. 8, pp. 2247-2259, Aug. 2016.

[127] M. Pan, M. Li, P. Li, and Y. Fang, Spectrum Trading in Multi-Hop Cognitive Radio Networks (SpringerBriefs in Electrical and Computer Engineering). New York, NY, USA: Springer, 2015. 
[128] M. Dong, G. Sun, X. Wang, and Q. Zhang, "Combinatorial auction with time-frequency flexibility in cognitive radio networks," in Proc. IEEE INFOCOM, Orlando, FL, USA, Mar. 2012, pp. 2282-2290.

[129] X. Feng, P. Lin, and Q. Zhang, "FlexAuc: Serving dynamic demands in a spectrum trading market with flexible auction," IEEE Trans. Wireless Commun., vol. 14, no. 2, pp. 821-830, Feb. 2015.

[130] G. Sun et al., "Coalitional double auction for spatial spectrum allocation in cognitive radio networks," IEEE Trans. Wireless Commun., vol. 13 , no. 6, pp. 3196-3206, Jun. 2014.

[131] F. Wu and N. Vaidya, "A strategy-proof radio spectrum auction mechanism in noncooperative wireless networks," IEEE Trans. Mobile Comput., vol. 12, no. 5, pp. 885-894, May 2013.

[132] I. Sugathapala, I. Kovachevic, B. Lorenzo, S. Glisic, and Y. Fang, "Quantifying benefits in a business portfolio for multi-operator spectrum sharing," IEEE Trans. Wireless Commun., vol. 14, no. 12, pp. 6635-6649, Dec. 2015.

[133] M. Li et al., "Optimal scheduling for multi-radio multi-channel multi-hop cognitive cellular networks," IEEE Trans. Mobile Comput., vol. 14, no. 1, pp. 139-154, Jan. 2015.

[134] N. Wang, E. Hossain, and V. K. Bhargava, "Backhauling 5G small cells: A radio resource management perspective," IEEE Wireless Commun., vol. 22, no. 5, pp. 41-49, Oct. 2015.

[135] M. J. Khabbaz, C. M. Assi, and W. F. Fawaz, "Disruption-tolerant networking: A comprehensive survey on recent developments and persisting challenges," IEEE Commun. Surveys Tuts., vol. 14, no. 2, pp. 607-640, 2nd Quart., 2012.

[136] A. Asadi, Q. Wang, and V. Mancuso, "A survey on device-to-device communication in cellular networks," IEEE Commun. Surveys Tuts., vol. 16, no. 4, pp. 1801-1819, 4th Quart., 2014.

[137] L. Wei, R. Q. Hu, Y. Qian, and G. Wu, "Enable device-to-device communications underlaying cellular networks: Challenges and research aspects," IEEE Commun. Mag., vol. 52, no. 6, pp. 90-96, Jun. 2014.

[138] L. Wei, A. Papathanassiou, Q. C. Li, and G. Wu, "System-level simulations for multi-hop D2D communications overlay LTE networks," in Proc. Int. Conf. Comput. Netw. Commun. (ICNC), 2016, pp. 1-5.

[139] Y. Ran, "Considerations and suggestions on improvement of communication network disaster countermeasures after the Wenchuan earthquake," IEEE Commun. Mag., vol. 49, no. 1, pp. 44-47, Jan. 2011.

[140] M. Casoni et al., "Integration of satellite and LTE for disaster recovery," IEEE Commun. Mag., vol. 53, no. 3, pp. 47-53, Mar. 2015.

[141] R. C. Shah, S. Roy, S. Jain, and W. Brunette, "Data MULEs: Modeling and analysis of a three-tier architecture for sparse sensor networks," $A d$ Hoc Netw., vol. 1, nos. 2-3, pp. 215-233, 2003.

[142] G. Chiarini, P. Ray, S. Akter, C. Masella, and A. Ganz, "mHealth technologies for chronic diseases and elders: A systematic review," IEEE J. Sel. Areas Commun., vol. 31, no. 9, pp. 6-18, Sep. 2013.

[143] A. Solanas, A. Martinez-Ballesté, P. A. Perez-Martínez, A. F. D. L. Peña, and J. Ramos, "m-Carer: Privacy-aware monitoring for people with mild cognitive impairment and dementia," IEEE J. Sel. Areas Commun., vol. 31, no. 9, pp. 19-27, Sep. 2013.

[144] B. M. C. Silva, J. J. P. C. Rodrigues, I. M. C. Lopes, T. M. F. Machado, and L. Zhou, "A novel cooperation strategy for mobile health applications," IEEE J. Sel. Areas Commun., vol. 31, no. 9, pp. 28-36, Sep. 2013.

[145] G. D. Clifford and D. Clifton, "Wireless technology in disease management and medicine," Аnпи. Rev. Med., vol. 63, no. 1, pp. 479-492, Feb. 2012.

[146] H. Lin, J. Shao, C. Zhang, and Y. Fang, "CAM: Cloud-assisted privacy preserving mobile health monitoring," IEEE Trans. Inf. Forensics Security, vol. 8, no. 6, pp. 985-997, Jun. 2013. 